\title{
Mechanical Regulation of Epithelial Tissue Homeostasis
}

\author{
Sara Kaliman, ${ }^{1, *}$ Maxime Hubert $\odot,{ }^{1, *,}$ Carina Wollnik, ${ }^{2}$ Lovro Nuić, ${ }^{3}$ Damir Vurnek, ${ }^{1,4}$ Simone Gehrer, ${ }^{1}$ \\ Jakov Lovrić@ ${ }^{3,1}$ Diana Dudziak, ${ }^{4,5,6,7}$ Florian Rehfeldt $\odot^{2,8, \sharp}$ and Ana-Sunčana Smith $\oplus^{1,3,8}$ \\ ${ }^{1}$ PULS Group, Department of Physics and Interdisciplinary Center for Nanostructured Films, \\ Friedrich-Alexander University of Erlangen-Nürnberg, 91058 Erlangen, Germany \\ ${ }^{2}$ University of Göttingen, Third Institute of Physics-Biophysics, 37077 Göttingen, Germany \\ ${ }^{3}$ Group for Computational Life Sciences, Division of Physical Chemistry, Ruđer Bošković Institute, \\ 10000 Zagreb, Croatia \\ ${ }^{4}$ Laboratory of Dendritic Cell Biology, Department of Dermatology, \\ Friedrich-Alexander University of Erlangen-Nürnberg, \\ University Hospital Erlangen, 91052 Erlangen, Germany \\ ${ }^{5}$ Deutsches Zentrum Immuntherapie (DZI), 91054 Erlangen, Germany \\ ${ }^{6}$ Comprehensive Cancer Center Erlangen - European Metropolitan Area of Nuremberg (CCC ER-EMN), \\ 91054 Erlangen, Germany \\ ${ }^{7}$ Medical Immunology Campus Erlangen, 91054 Erlangen, Germany \\ ${ }^{8}$ University of Bayreuth, Experimental Physics 1, 95440 Bayreuth, Germany
}

(Received 27 August 2020; revised 18 March 2021; accepted 26 May 2021; published 5 August 2021; corrected 18 August 2021)

\begin{abstract}
Despite recent efforts to understand homeostasis in epithelial tissues, there are many unknowns surrounding this steady state. It is considered to be regulated by mechanoresponse, but unlike for single cells, this idea remains heavily debated for tissues. Here, we show that changes in matrix stiffness induce a nonequilibrium transition from tubular to squamous Madin-Darby Canine Kidney II tissues. Nonetheless, despite different cell morphologies and densities, all homeostatic tissues display equivalent topologies, which, hence, must be actively targeted and regulated. On the contrary, the mechanoresponse induces dramatic changes in the large-scale organization of the colonies. On stiff gels, this yields an unreported cooperative state of motile cells displaying higher densities than in the arrested homeostatic state, which suggests a more complex relation between cell density and motility than previously anticipated. Our results unequivocally relate the mechanosensitive properties of individual cells to the evolving macroscopic structures, an effect that could be important for understanding the emergent pathologies of living tissues.
\end{abstract}

DOI: $10.1103 /$ PhysRevX.11.031029

Subject Areas: Biological Physics, Soft Matter

\section{INTRODUCTION}

Homeostasis [1] denotes maintenance of the morphology and metabolic functions in differentiated tissues. It is preceded by tissue growth or regeneration in which complex tissue architecture emerges on multiple length scales [2]. It spans from the size of a cell, to the mesoscopic level with structures such as crypts [3], to the macroscopic

\footnotetext{
These authors contributed equally to this work.

Corresponding author.

maxime.hubert@fau.de

*Corresponding author.

florian.rehfeldt@uni-bayreuth.de

${ }^{\S}$ Corresponding author.

smith@physik.fau.de

Published by the American Physical Society under the terms of the Creative Commons Attribution 4.0 International license. Further distribution of this work must maintain attribution to the author(s) and the published article's title, journal citation, and DOI.
}

formation of entire organs. In epithelium, major strategies of homeostatic control are contact inhibition of proliferation [4] and locomotion [5], as well as cell extrusions [6], which are all dependent on the actual cell density. As such, the homeostatic steady state $\mathrm{H}_{\mathrm{PL}}$ (inhibition of proliferation and locomotion), with its characteristic structure, is cooperative by nature [7] and a result of nonequilibrium selforganization, a process that has not been fully understood so far. In the biomedical context, however, the homeostatic states, with their characteristic cell densities and shapes, have been very well characterized. Consequently, deviations from typical phenotypes, both on the cellular and compartment levels, are often considered important in the diagnosis of a broad range of diseases [8]. Nonetheless, the emergent tissue properties, as well as the biochemical and biophysical regulations of the homeostatic steady state, are still subject to intense research [9-12].

Besides the complex biochemical signaling sequences involved in homeostasis [13], the $\mathrm{H}_{\mathrm{PL}}$ architecture is tightly regulated by physical forces that are generated within and 
between the cells, as well as by the interactions with the extracellular matrix (ECM), as part of mechanoresponse $[14,15]$. In individual adherent cells, the mechanosensitive force balance is strongly coupled with the cell shape, motility, nuclear positioning, and many other processes [10,16-21]. During development, mechanoresponse is crucial for the compartmentalization of tissues and the development of organs. For example, the control of human epiblast and amnion development was found to be partly determined by the mechanical properties of the nichelike environment $[22,23]$. In homeostatic tissues, however, the role of mechanosensing is still unclear. To the contrary, there is growing evidence that a number of pathological conditions involve changes in stiffness $[17,24,25]$ and viscosity $[26,27]$ of the extracellular matrix. In cancer, for example, matrix stiffening has been related to the epithelial-mesenchymal (a)

(c)
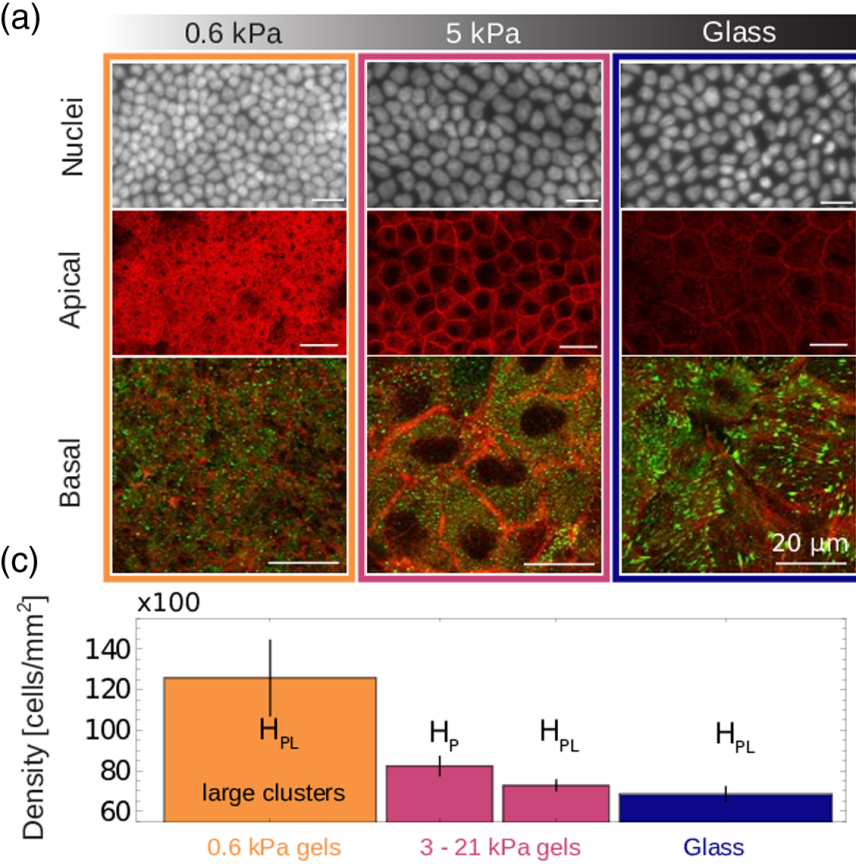

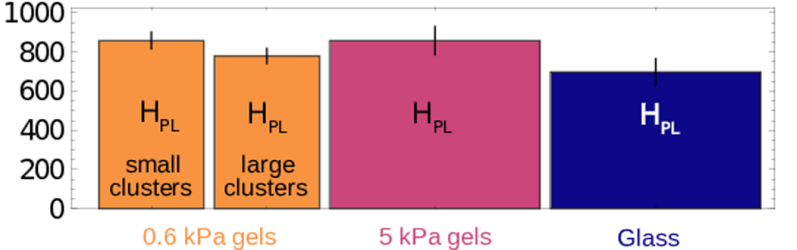

(b)
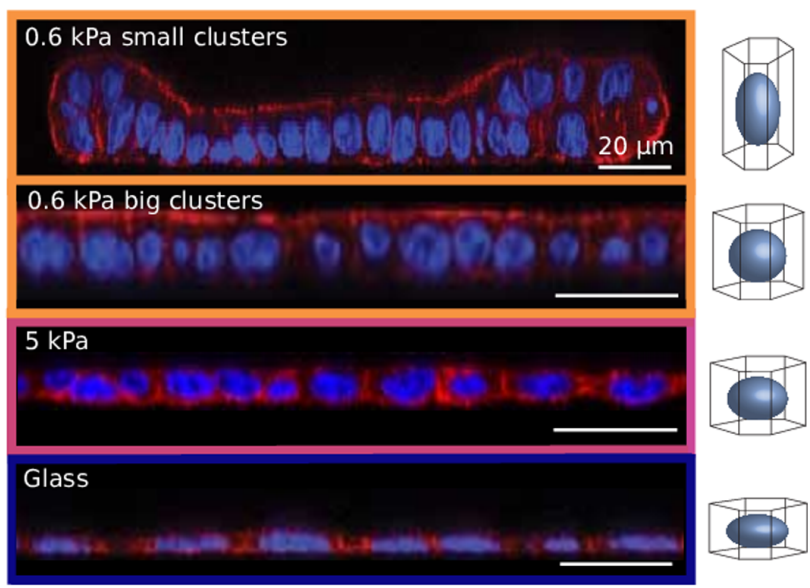

(d)

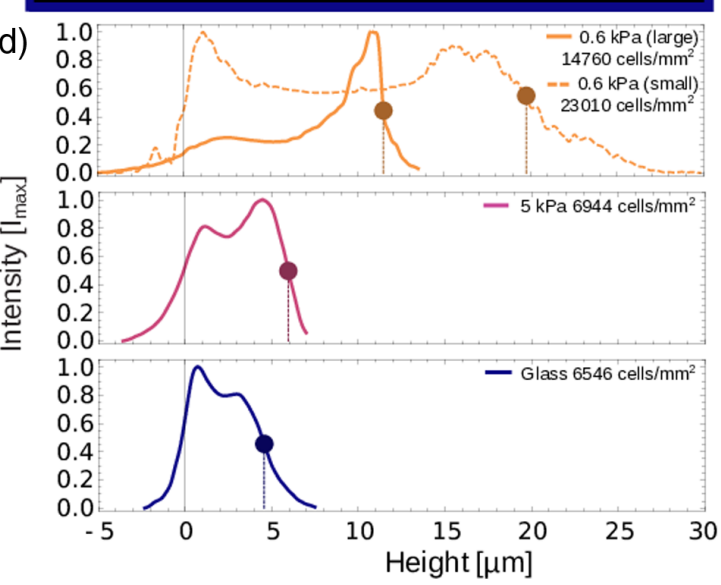

FIG. 1. Characteristics of the homeostatic state of the tissue as a function of the substrate stiffness. Tissues are grown on soft gels (orange colored frames and lines), hard gels (pink), and on glass (blue). (a) Characteristic confocal images in the plane of the monolayers fixed early in the fourth day after seeding. The top panel presents Hoechst stained cell nuclei. In the middle panel, apical phalloidin stained actin is imaged. The bottom panel shows paxillin stained using secondary antibodies in green and actin in red to visualize focal adhesions. (b) Reconstructed slice through the monolayers showing columnar MDCK-II tissue on soft gels, cuboidal tissue on hard gels, and squamous tissue on glass substrates. Actin is shown in red and nuclei in blue. Small colonies on soft gels are characterized by a monolayer area smaller than $2.8 \times 10^{-2} \mathrm{~mm}^{2}$, and finite-size effects still play a role. Larger colonies are imaged away from the edge. (c) Bar charts of the average cell density (top) and volume (bottom). Error bars indicate standard deviations from measurements in different colonies grown under identical conditions. This deviation is, however, similar to standard deviations in density within a single colony (see Appendix B for details). Unlike cell volumes in different tissues, cell density shows statistically significant differences even between hard gels and glass. However, the error in the measurement of the volume, obtained from the propagation of uncertainties in measuring density and height, is of the order of $10 \%$. Here, density was calculated from approximately $500 \times 10^{3}$ cells at $0.6 \mathrm{kPa}$, from $208 \times 10^{3}$ cells grown in tissues on substrates with stiffness between 3 and $21 \mathrm{kPa}$, and from $27 \times 10^{3}$ cells on glass (see Appendix B 2). The height was calculated for 100 (glass) to 350 cells (soft gels) depending on the density considered. The notations $\mathrm{H}_{\mathrm{PL}}$ and $\mathrm{H}_{\mathrm{P}}$ stand for the homeostatic state with inhibition of proliferation and locomotion and for the homeostatic state with inhibition of proliferation, respectively. (d) Average intensity of the stained actin as a function of the distance from the substrate. The height of the monolayer is determined as the difference in heights between the two inflection points in the intensity curves. For simplicity, all inflection points at the basal surface are co-aligned. The circles indicate the apical surface. The density in the presented segment where the volume is measured is indicated in each graph. 
transition and the significant increase of cell motility yielding metastasis [28]. However, mechanistic studies in model systems, involving the modulation of matrix stiffness, provided contradictory evidence regarding the properties and the topology of the $\mathrm{H}_{\mathrm{PL}}$ steady state, the shapes of constitutive cells, as well as the macroscopic self-compartmentalization in growing colonies [29-36].

In order to resolve this debate and provide a deeper insight regarding the influence of cell mechanosensitivity at the microscopic and macroscopic levels, we perform here a systematic study of the effects of mechanosensing on the growth, self-assembly, and homeostasis of a model epithelium. We take advantage of a fully controlled, biomimetic environment to clearly delineate the mechanoresponse on different length scales and timescales, demonstrating that both the shapes of cells and the compartmentalization of tissue during development respond to changes in the mechanical properties of the environment. Interestingly, however, we find that the topology of the homeostatic tissue is independent of the stiffness, despite variations in density.

\section{MECHANORESPONSE OF CELLS WITHIN THE HOMEOSTATIC TISSUE}

We grow Madin-Darby Canine Kidney II (MDCK-II) tissues on substrates with systematically varying stiffness. The substrates are glass or polyacrylamide (PA) gels with an elastic Young's modulus $E=0.6,3,5,11$, and $21 \mathrm{kPa}$ ( $\delta E= \pm 0.3 \mathrm{kPa}$, see the Appendix A for methodological details) covalently and uniformly coated with collagen-I. This change in substrate stiffness should result in different stress-generation patterns, hence altering the tissue's steady state. To sample sufficient statistics on different length scales, we produce centimeter-wide monolayers, starting from a droplet containing approximately $3 \times 10^{4}$ cells. All colonies are grown for 4-6 days in controlled conditions, upon which the homeostatic state $\mathrm{H}_{\mathrm{PL}}$ is fully formed in the central selfassembled compartment of the tissue (see Figs. 6 and 7). In $\mathrm{H}_{\mathrm{PL}}$, if any proliferation occurs, it is balanced by the apoptosis rate, such that the mean density no longer depends on time. Furthermore, no net locomotion is observed (see Fig. 6).

Initially, we verify the mechanosensitivity of cells in homeostasis by imaging the subcellular cytoskeletal and adhesion structures as shown in Fig. 1(a) (for technical details and the biological function of stained proteins, see Appendix A 2). As reported for single cells, increasing the substrate rigidity yields larger focal adhesions and stronger actomyosin stress fibers [21] but weaker apical tension [Fig. 1(a)] as evidenced by the modulation of apical and basal peaks in the actin vertical density profile [Fig. 1(d)].

On glass, the cell-substrate adhesion energy dominates, with the actin cytoskeleton [14,37] concentrating on the basal surface [Fig. 1(d)]. The result is the appearance of squamous cells (height $h=4.6 \pm 0.4 \mu \mathrm{m}$, height-to-base length $h / r=0.35 \pm 0.04)$ with a homeostatic density
$\rho\left(\mathrm{H}_{\mathrm{PL}}\right)=6860 \pm 360$ cells $/ \mathrm{mm}^{2} \quad$ [Fig. $\left.1(\mathrm{c})\right] . \quad$ Under these conditions, the basal tension is given by the formula from Hannezo et al. [14], which reads $\left|\gamma_{b}\right|=$ $\sqrt{3} \alpha r^{2} / V^{2} \approx 5.988 \times 10^{-4} \mathrm{~N} / \mathrm{m}$ with $V$ the cell volume and $\alpha \approx 10^{-24} \mathrm{Jm}^{2}$, the latter being the confinement energy of the cytoplasmic components.

On hard gels, the actin distribution displays both basal and apical maxima, indicating weakening of adhesion and strengthening of the apical tension [Fig. 1(d)]. Consequently, the tissue becomes cuboidal $(h=5.9 \pm 0.5 \mu \mathrm{m}$, $h / r=0.47 \pm 0.05)$. Surprisingly, the density of the homeostatic state $\rho\left(\mathrm{H}_{\mathrm{PL}}\right)=7280 \pm 260$ cells $/ \mathrm{mm}^{2}$ is slightly but consistently larger than on glass, yet statistically, it is the same on gels of stiffness in the range of $3 \mathrm{kPa}$ to $21 \mathrm{kPa}$ (see Table I). This trend is systematic over different series of experiments, although the averages on glass and gels may differ by a few hundred cells per square micron, consistent with the differences noticed previously [38].

On soft gels, most actin is within the apical belt [Figs. 1(b) and 1(d)] to mediate a strong contractile tension $\Lambda_{\alpha}$, estimated by Hannezo et al. [14] to be $\Lambda_{\alpha}=2 \alpha /\left(\sqrt[3]{9 V} r^{2}\right)=$ $1.491 \times 10^{-9} \mathrm{~N}$. The emergent density is high $\rho\left(\mathrm{H}_{\mathrm{PL}}\right)=$ $12580 \pm 1860$ cells $/ \mathrm{mm}^{2}$ and accompanied by a phase transition toward a columnar morphology $(h=11.5 \pm$ $0.6 \mu \mathrm{m}, h / r=1.20 \pm 0.15)$. To accommodate this, cell nuclei become highly asymmetric, with the major axis perpendicular to the substrate [Fig. 1(b)].

Furthermore, all homeostatic states have the same average cell volumes within the accuracy of the measurements [Fig. 1(c)]. We can therefore conclude that cells in $\mathrm{H}_{\mathrm{PL}}$ sense the stiffness of the underlying substrate by cooperatively adjusting their cell morphology as well as their nuclei shape and orientation. This process is enabled by the modulations in adhesiveness and changes in the spatial distribution of actin.

\section{UNIVERSAL TOPOLOGY OF HOMEOSTATIC STATES}

We further investigate the organization of cells building the homeostatic states from a statistical perspective (technical details are given in Appendix C). Rather than extracting the morphological properties of cells directly from segmented images of the cell plasma membrane as shown in Fig. 2(a), which can be challenging, we approximate the cell shape using the set-based Voronoi tessellation, referred to as SVT in the remainder of this article. This method allows for generating very large and robust data sets as shown in Fig. 9. It relies on accurate segmentation of nuclei, which we achieve with our home-built posttreatment algorithm with an accuracy of $99 \%$, even at the highest tissue densities [38]. As discussed in more detail in Appendix C 1, the accuracy of the SVT at the densities typically achieved for the $\mathrm{H}_{\mathrm{PL}}$ states is high. The difference from direct segmentation of the membrane does not exceed 
(a)

$$
0.6 \mathrm{kPa}
$$
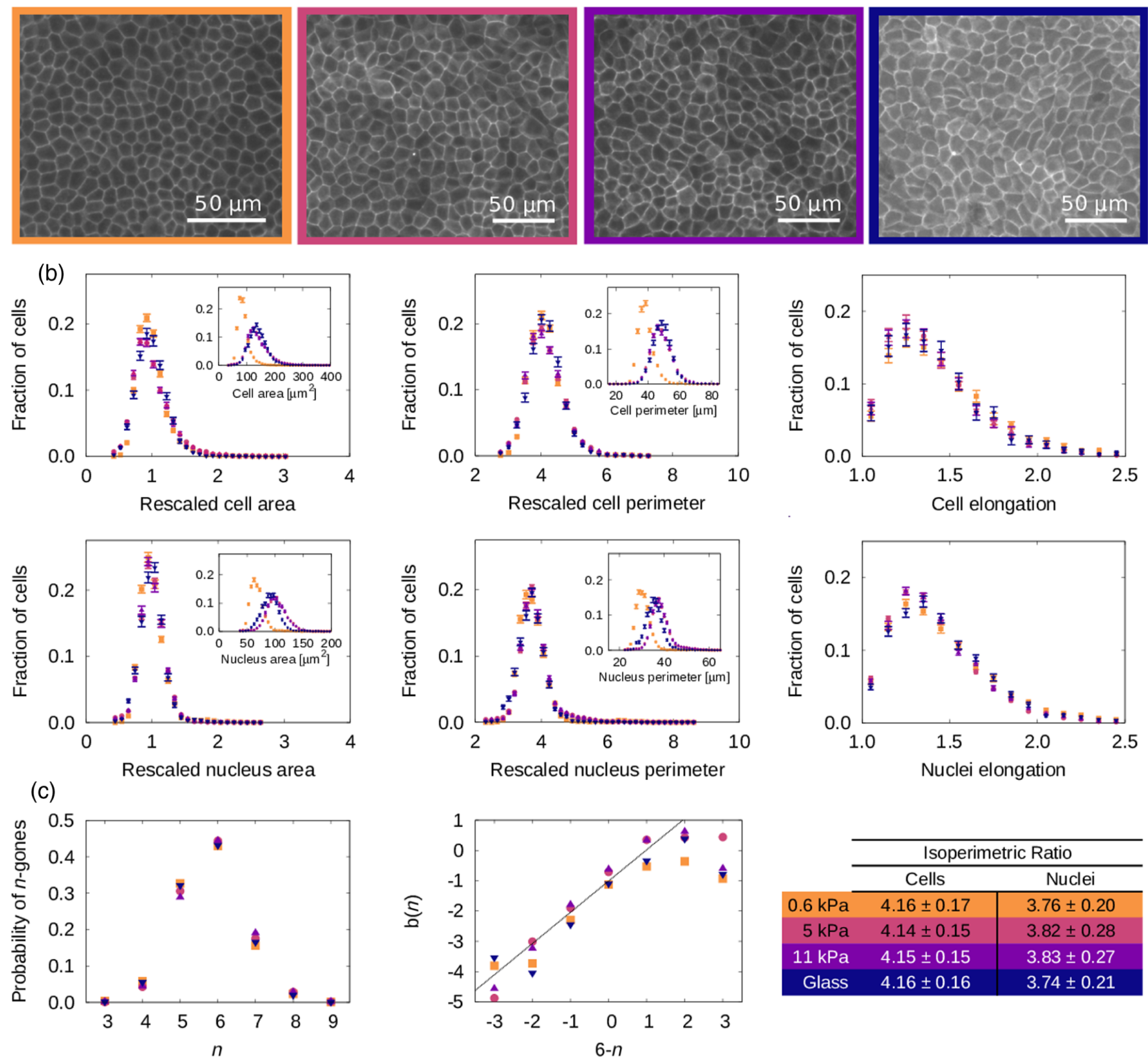

FIG. 2. Structure and topology of tissues in the homeostatic state. (a) Images of $\beta$-catenin stained cell membranes in the steady state $\mathrm{H}_{\mathrm{PL}}$ (inhibited for proliferation and locomotion). The magnification in different frames is chosen such that cells appear equally large, to visualize the morphological rescaling. (b) Distributions of morphological measures of cells obtained through set-based Voronoi tessellations (top row) and nuclei (bottom row). For soft gels (orange) 4257 cells are extracted from large colonies to avoid finite-size effects. For tissues grown on $5 \mathrm{kPa}$ (pink), $11 \mathrm{kPa}$ (purple), and glass (blue) substrates, 9095, 5244, and 2575 cells are considered. The distributions of rescaled measures (each length divided by the square root of the mean area) are provided as main graphs, while the insets show the original data. Distributions for area (left), perimeters (middle), and elongations (right) are shown. Elongations are extracted by calculating the principle axis of inertia of the relevant object (cell or nucleus) and finding the ratio. Error bars correspond to the standard deviation on 50 independent subsets of cells (see Appendix C). (c) Conservation of topological measures relative to tissues grown on different substrates. The color code is consistent throughout the figure. Distribution of cells with $n$ sides (i.e., neighbors) is on the left, while, in the center, the Aboav-Weaire's law is measured through the parameter $b(n)=\langle m(n)\rangle n-6 n-\sigma_{n}^{2}=\gamma(6-n)$. The isoperimetric ratios for the average cell and nuclei shapes are provided in the table on the right. 
$10 \%$ for any measure, even when compared to a reference set extracted directly from high-accuracy methods that do not support massive tissue imaging [38]. Using this approach, we obtain distributions of cell and nuclei area, perimeter, and elongation, as shown in the insets of Fig. 2(b).

For the purpose of establishing a unique metric between various tissues, all lengths are rescaled by the square root of the average cell area $\langle A\rangle$ in a particular tissue. This process indeed leads to the collapse of data such that the distributions of area, perimeter, and elongation for both cells and nuclei show no appreciable differences, as highlighted by the overlapping error bars [Fig. 2(b)]. Actually, the differences between two distributions of different substrates are comparable to differences between distributions generated from large subsets of cells obtained from the same substrate, which implies that the average cell and the deviations from an average cell are, within the statistical accuracy of the data, self-similar among all tissues. Furthermore, all of the statistics driven from those distributions are within the accuracy of the measurements. The same conclusion holds for the cell nuclei, which is the distribution imposing the cell shapes in the plane of the monolayer.

Furthermore, to analyze the connectivity of the cells within the $\mathrm{H}_{\mathrm{PL}}$ compartments of the tissues, we calculate the distributions of the number of neighbors $n$ of all cells [Fig. 2(c)]. Similarly to the case of morphological measures, we find that these distributions are statistically equal in all sets and insensitive to the substrate stiffness and, therefore, the cell density. Furthermore, these distributions are very similar to previously reported ones in different epithelium [39]. While the most common cell shape in all $\mathrm{H}_{\mathrm{PL}}$ tissues is hexagonal (approximately 45\%), the distributions of $n$ are positively skewed. Actually, the fractions of cells with five and seven neighbors are significant, with a larger pentagon component (30\% pentagon compared to $20 \%$ heptagon). This asymmetry exists already in the random packings of ellipses at densities and elongations comparable to the nuclei density and shapes [40], albeit, in tissues, the disparity in the fractions of pentagons and heptagons is significantly larger.

Finally, to explore the topology of the tissues, we analyze the correlations between the number of neighbors of cells with $n$ sides and the average number of neighbors $m(n)$ that cells adjacent to ones with $n$ sides have. When this relation is linear, it is known as the Aboav-Weaire's law [41-43], which states that

$$
\langle m(n)\rangle=6-\gamma+\left(6 \gamma+\sigma_{n}^{2}\right) / n .
$$

Here, $\sigma_{n}^{2}$ is the second central moment of the distribution of $n$, and $\gamma$ is a constant that may decrease as $\sigma_{n}^{2}$ increases $[44,45]$, or may be independent of $\sigma_{n}^{2}$ [46]. This relation has been commonly used to explore the topology of tessellations and arrangements in cellular assemblies [40,47,48].
In the case of homeostatic MDCK-II tissues, irrespective of the conditions in which they were grown, all tissues show the same linear dependence [Fig. 2(c)], which suggests that cells with fewer neighbors tend to have neighbors with more sides, and vice versa. This trend is violated for cells with more than eight or less than four neighbors. Here, the linear relation also shows a clear offset along the $y$ axis. This offset suggests that the topology of the homeostatic MDCK-II, which emerges from the geometry of the spatial distribution of nuclei, is actively maintained to achieve a particular connectivity.

The relation between geometry and topology can be further explored by calculating the correlations between the number of neighbors $n$ and the average rescaled area $\left\langle A_{n}\right\rangle$ and rescaled perimeter $\left\langle P_{n}\right\rangle$ of a cell with $n$ neighbors. These linear relations are known as the Lewis law [49-52] and Desh law [53], respectively. They suggest that large cells have a tendency to have more neighbors, while, inversely, small cells have a tendency to have less neighbors. Indeed, these linear relationships are also confirmed in MDCK-II tissues (see Fig. 10), suggesting, again, that geometrical elements, as emphasized by random packings [40], remain important in tissues, even though the cell and nuclei shape distributions are strongly regulated in the homeostatic state.

We thus conclude that tissue topology is the key property actively maintained in homeostasis, leading to a well-defined spatial organization, which is, by and large, universal for all MDCK-II steady states. Universality of the topology is also reflected in the equivalence of the isoperimetric ratios of cells [54] and of cell nuclei, which remain independent of the matrix stiffness [table in Fig. 2(c)]. This suggests that all homeostatic MDCK-II tissues are in mechanically equivalent steady states. The latter is, however, governed by very different contributions to the force balance, as reflected in different cells' three-dimensional shapes and densities.

\section{MACROSCOPIC EFFECTS OF MECHANORESPONSE IN TISSUES GROWN ON HARD GELS AND GLASS}

Finally, to bring additional insights about the role of mechanoresponse on the macroscopic scale (technical details are given in Appendix D), we investigate the structure of tissues supporting the homeostatic state. On glass and on hard gels, colonies are radially symmetric [Fig. 3(a)], and the $\mathrm{H}_{\mathrm{PL}}$ state develops in the center [flat segment of the density profiles in top panels of Fig. 3(b)].

With increasing radius, after the $\mathrm{H}_{\mathrm{PL}}$ state and towards the tissue's outer rim, three more compartments are reported. We designate these compartments $C_{1}, C_{2}$, and $C_{3}$. The large $C_{1}$ compartment is characterized by cells that become motile and divide, and their density continuously drops until the $C_{2}$ compartment. The appearance of the latter is characterized by a change of curvature of the density profile [Figs. 3(b), 3(c), and 3(e)]. In $C_{2}$, the cell density increases, while the basal actin and paxillin show a 
(a)
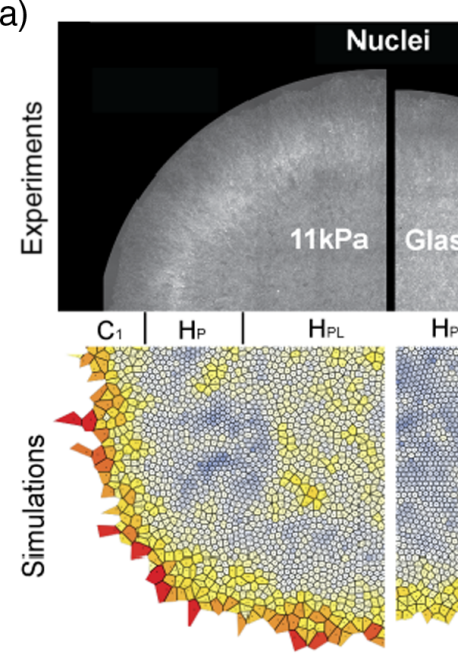

(c)

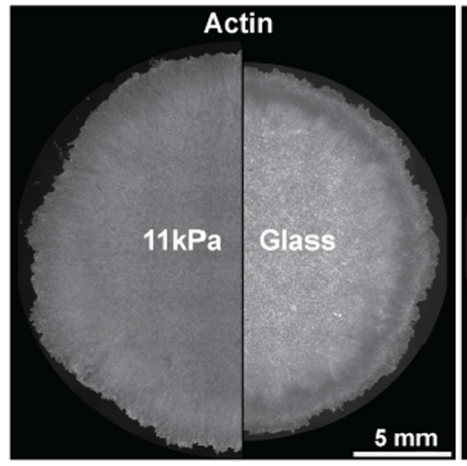

(e)

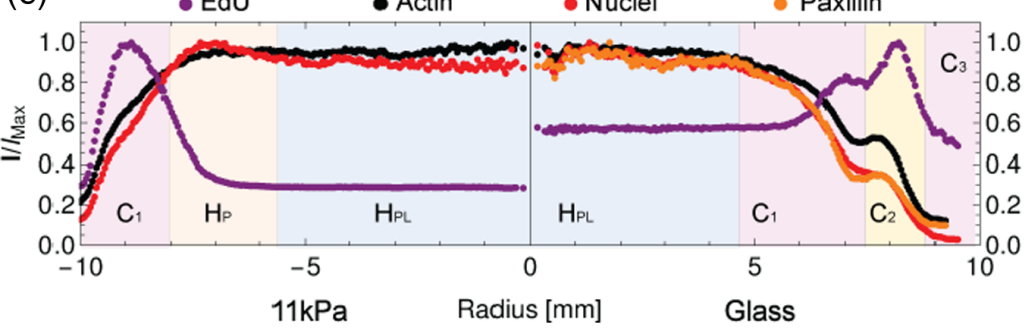

(b)

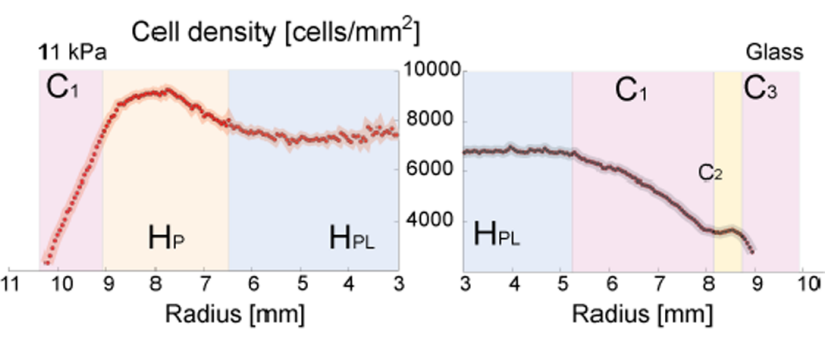

Cell density $[\mathrm{H}\llcorner\mathrm{L}]$
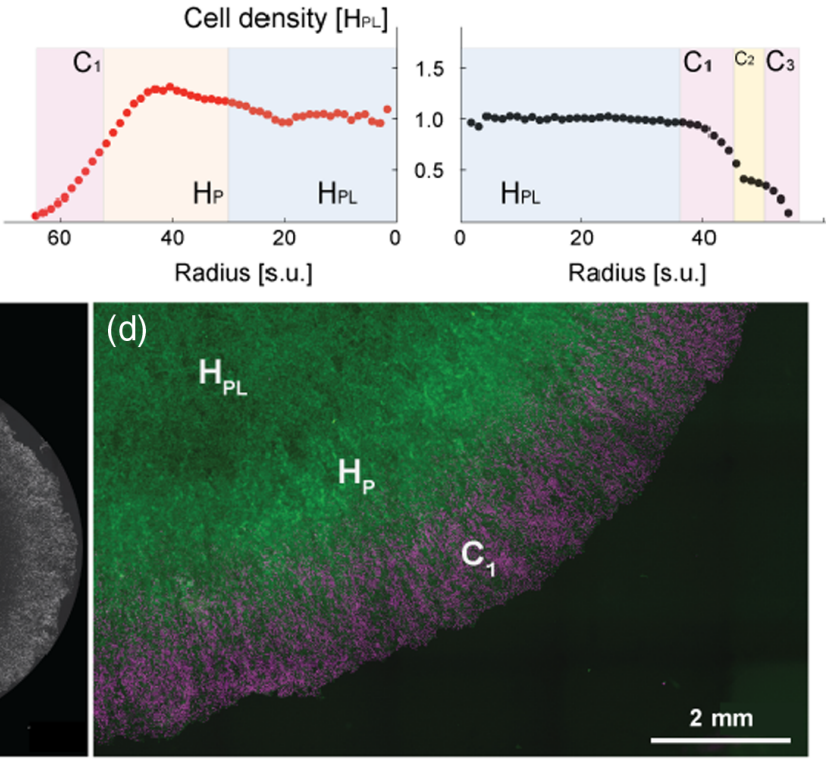

(f)

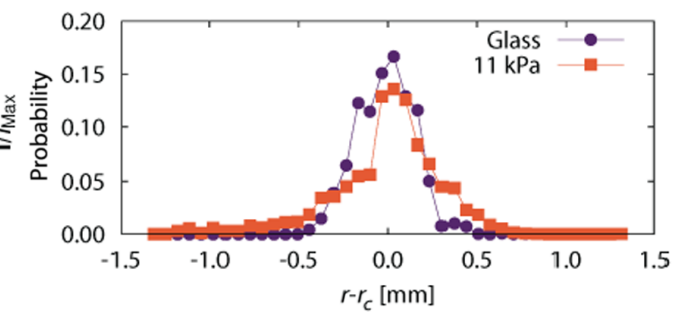

FIG. 3. Macroscopic organization of the tissue surrounding the homeostatic state on hard gels and glass. $\mathrm{H}_{\mathrm{PL}}$ and $\mathrm{H}_{\mathrm{P}}$ stand for the homeostatic state with inhibition of proliferation and locomotion and for the homeostatic state with inhibition of proliferation, respectively. Additional compartments are denoted with $C_{n}$, as defined in the main text. (a) Hoechst stained cell nuclei throughout the tissues grown for 6 days (top panel) are compared to simulations (bottom panel). Colonies on hard gels (left panel) are compared to ones developed on glass substrates (right panel). The scale bar indicates $2 \mathrm{~mm}$. Various compartments are indicated in between the simulation and experimental diagrams. (b) Experimental density profiles obtained from the linear relation between density and light intensity (top row) on gels (red curve on the left) and glass (black curves on the right); more details are given in Appendix D 1. Simulation counterparts are shown in the bottom row. Parameters are given in Table III, with $f_{0}=64$ and $\Gamma=0.5$ and 2.0 for glass and hard gels, respectively. All values are identical for all cells; the macroscopic compartment they are in does not influence the parameters. (c) Experimental cell clusters on hard gels and glass substrates stained for actin and EdU, showing only residual proliferation in the $\mathrm{H}_{\mathrm{PL}}$ state. (d) Segment of a tissue grown on hard gels showing no proliferation within the $\mathrm{H}_{\mathrm{PL}}$ and $\mathrm{H}_{\mathrm{P}}$ compartments. The purple, EdU stained nuclei are overlayed with the Hoechst stain in green. (e) Intensity profiles for actin (black), nuclei (red), paxillin (orange, only on glass), and EdU (purple) through the colonies grown on hard $11 \mathrm{kPa}$ gels and glass. (f) Tissue roughness evaluated by the normalized distributions of the deviations of the edge from the average shape.

stronger signal compared to the neighboring $C_{1}$ and edge $C_{3}$ compartments. If one assumes that the intensity of basal paxillin (and actin) relates to the size of focal adhesions, this result suggests stronger cell adhesiveness in the $C_{2}$ compartment overall, compared to $C_{1}$ and $C_{3}$. Com- partment $C_{3}$ consists of a large number of cells that transiently adopt the phenotype of leader cells [55] with extended lamellipodia (see Fig. 15). Cells in $C_{3}$ commonly exchange their neighborhood, while the average speed of cells saturates (see Fig. 13). 
We capture this compartmentalization using dissipativeparticle-dynamics (DPD) simulations [56-58] (technical details are given in Appendix E, with parameter values summarized in Table III). The DPD simulations integrate various contributions in the effective terms in a similar way as the theory for the cell shape [14]. As such, the substrate stiffness is accounted for through the friction associated with propulsion onto the substrate. The later contribution is a combination of adhesion and traction forces, which are indeed substrate dependent. To obtain the compartmentalization as seen on glass, the observation of the saturation of the cell velocity at the edge of the colony (see Fig. 13) is incorporated into the model (see Fig. 17). Without this assumption, $C_{2}$ and $C_{3}$ cannot be delineated with the basic model. However, imposing the saturation of cell velocities at the edge in $C_{3}$, we find that $C_{2}$ spontaneously appears [bottom right panel in Fig. 3(b)]. In turn, this can mechanically stabilize $C_{1}$ during the spreading of the colony.

A different internal structure of the colony has been observed in tissues grown on hard gels. While not displaying the $C_{2}$ and $C_{3}$ compartments, the colony still possesses an outer compartment similar to $C_{1}$ and extending all the way to the edge. On hard gels, only the first outer layer of cells is decorated with the leading cell phenotype (see Fig. 15). Furthermore, we find that large-scale roughness (see Appendix D4) is more important on hard gels compared to glass, where roughness emerges mostly from small deviations from the average shape [Fig. 3(f)]. This case is consistent with frequent and fast changes of the neighborhood in the $C_{3}$ compartment on glass. On gels, on the contrary, large deviations from the average circular shape have time to develop since the restructuring of the very edge is slower.

The key new feature in tissues grown on hard gels is the new compartment denoted as $\mathrm{H}_{\mathrm{P}}$ [Figs. 3(a) and 3(c)-(e)], in which cell proliferation is inhibited [Figs. 3(c)-3(e)] but the locomotion is still ongoing (see Fig. 12). Surprisingly, the cell density in $\mathrm{H}_{\mathrm{P}}$ is significantly higher than in the already-discussed, fully arrested $\mathrm{H}_{\mathrm{PL}}$ homeostatic state [Figs. 1(c) and 3(b)]. This result is unexpected given the usually reported inverse relation between tissue density and average cell velocity [59]. Note that the $\mathrm{H}_{\mathrm{P}}$ compartment can also be seen in the actin signal as shown in Figs. 3(c) and 3(e). Furthermore, since $\mathrm{H}_{\mathrm{PL}}$ and $\mathrm{H}_{\mathrm{P}}$ display statistically equivalent topologies, this result shows that there could be a more complex relation between tissue structure and motility than previously reported (see Fig. 10).

To further investigate the origin of this highly cooperative state, we use DPD simulations and show that similar behavior appears if a surplus active pressure is generated at the outer edge of $\mathrm{H}_{\mathrm{PL}}$ [Fig. 3(b)]. We rationalize this surplus active pressure by the higher speed of cells on soft substrates than on stiff ones (see Ref. [60] and references therein). Hence, we change the activity of the cells and increase the parameter $\Gamma$, which is the amplitude of the force produced by all cells with respect to the direction of the velocity vector; the value of this parameter is constant across the whole tissue. Its amplitude for the gels is thus set to $\Gamma=2.0$ s.u., compared to glass, where it has been chosen as $\Gamma=0.5$ s.u. Indeed, this higher pressure, given its definition, increases the cell speed. This sole difference is sufficient to produce the nonmonotonic density profile characteristic of hard gels. The density overshoot in the $H_{P}$ compartment therefore comes from the competition between activity and friction at the substrate interface. Consequently, the colonies on gels grow somewhat faster than on glass, which indeed seems to be the case even in experiments.

The growth is naturally supported by the proliferation of cells, which, by and large, dominates the $C_{1}$ compartment in gels and $C_{1}$ and $C_{2}$ on glass [Fig. 3(e)] but strongly decays toward the very edge of the colony in $C_{3}$. This observation suggests that the probability of division strongly couples to the cell size, yet the proliferation pressure in the colony is built up millimeters away from its edge. The force therefore comes from the core which extends the colony. This situation is different than the force that a nonadhesive interface applies pointing inwards and confining the colony [61]. The overall pressure is then spatially dependent and is built from the propensity of cells to divide and to move to establish a particular density profile.

In the $\mathrm{H}_{\mathrm{PL}}$ state, proliferation is small and often occurs at the position of $20-40 \mu \mathrm{m}$ large defects in the tissue (see Appendix F and Fig. 18). These defects are associated with extrusion events, which start to take place typically 4-5 days after seeding as the homeostatic state is established. In agreement with previous reports in the literature [62], these extrusions do not trigger the appearance of new colonies. Actually, as the tissue grows beyond the edges of the initial seeded drop, no secondary colonies are observed alongside the main one. Cell divisions thus act to heal the tissue. Given that the extrusions are significantly more common on glass than on gels, the residual EdU signal is also more intense. These defects are significantly different to "domes" [63], which are, however, significantly larger (average size of the order of $4000 \mu \mathrm{m}^{2}$ ). Domes appear as transient blisters when the homeostatic state is achieved (see Fig. 19), and cells start to perform their physiological role, pumping ions from the apical to the basal side of the tissue. Because of the nature of gels, pumped ions do not accumulate below the basal side of the tissue, and therefore, domes do not appear on the gels. However, the extrusions are common and a natural part of the maintenance of the homeostatic state.

\section{MACROSCOPIC ORGANIZATION ON SOFT GELS}

A fundamentally different mode of compartmentalizing the homeostatic state takes place for soft gels (stiffness of $0.6 \mathrm{kPa}$ ). Here, the cells first form a small spherical agglomerate and, over time, create the $\mathrm{H}_{\mathrm{PL}}$ monolayer in 

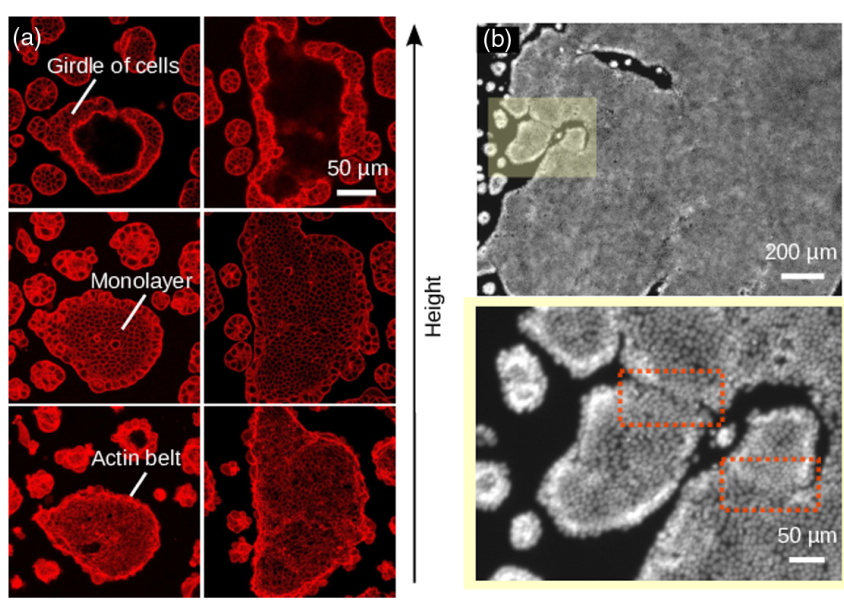

FIG. 4. Tissues grown on soft gels. (a) Actin stained cells within clusters grown on soft substrates $(E=0.6 \mathrm{kPa})$. Three confocal slices of the same colony are provided, focusing on the basal side with the actin belt (bottom), central monolayer (middle), and the multilayered girdle of cells (top). (b) Epifluorescence image of nuclei stained with Hoechst dye of a tissue grown on soft $0.6 \mathrm{kPa}$ gels. The yellow box in the top panel is enlarged in the bottom. The girdle and the monolayer can be clearly discerned. Spherical agglomerates, small colonies, and a segment of the large colony are shown simultaneously. In the bottom panel, linelike scars due to the merging of two colonies are boxed.

the center of the colony. Consistently with previous work [34], we find a critical minimum size of the microcolonies of $4.7 \times 10^{-3} \mathrm{~mm}^{2}$, at which the formation of the monolayer is observed in the central region, with densities up to 25000 cells $/ \mathrm{mm}^{2}$ [Fig. 1(b)]. As the monolayer spreads up to $2.8 \times 10^{-2} \mathrm{~mm}^{2}$, the density decreases until the finite density of about $12580 \mathrm{cell} / \mathrm{mm}^{2}$ is achieved in the central compartment of the colony [Fig. 4(b)], which suggests that finite-size effects have a range of about $40 \mu \mathrm{m}$ and spread over about 10 cells.

The columnar $\mathrm{H}_{\mathrm{PL}}$ state [Fig. 1(b)] is encircled only by a $40 \pm 10 \mu$ m-thick girdle of cells comprising a few cell layers [top panels in Fig. 4(a)]. Moreover, those girdle cells that are in contact with the substrate build a thick actin filament belt that surrounds the whole colony [bottom panels in Fig. 4(a)]. In small colonies, the actin belt is more circular, presumably mechanically stabilizing the central single-layered compartment. This compartmentalization into the girdle and the monolayer is maintained over all colonies. For larger clusters, the overall shape is irregular, and the actin belt may vary in thickness [Fig. 4(b)].

These highly irregular shapes of colonies emerge from the dynamics of growth and the formation of subcolonies following cell extrusions. Namely, on soft gels, functional cells are expelled as soon as the monolayer forms, leaving small scars often surrounded by a multilayered ring of cells that is subsequently filled (see Fig. 18). At the same time, small secondary colonies appear and are found throughout the experiment, up to 12 days. Extruded cells land near the mother colony and develop daughter colonies. As the colonies grow, they merge, contributing to the complex shape of the large colony. The merging events are associated with the restructuring of the girdles, which induces linelike defects of smaller monolayer densities [Fig. 4(b)]. Since all colonies clearly present only two compartments, we presume that the reorganization takes place immediately as the two unstructured multilayered compartments start to touch. Given that the locomotion is prohibited everywhere but in the proliferating girdle, this regime is associated with a very different growth dynamics [38], which should be explored in the future.

Finally, a coexistence of the two regimes of growth is observed on gels with a stiffness of $1.2 \mathrm{kPa}$ (see Fig. 8). Here, we observe the compartmentalization characteristic of soft and hard gels within a single colony. However, given the uncertainties in the gel stiffness at $0.6 \pm 0.3$ and $1.2 \pm 0.3 \mathrm{kPa}$, we cannot distinguish if the two coexisting states are the result of the fluctuations in the gel stiffness or a genuine coexistence of dynamic phases in a very narrow range of gel stiffness. Furthermore, specific consideration should be given to the interplay between mechanosensitivity and adhesiveness. Therefore, it would be interesting to systematically modulate the density of adhesion molecules on the surface, which could be done using existing protocols $[21,64,65]$. Manipulating the density of adhesive sites was found to be important in the case of single cells $[21,64]$ and could influence the position of the transition. Notably, however, it is interesting that the strongest mechanoresponse of MDCK-II tissues occurs exactly in this range. Namely, it is well established that there is a critical range of elasticity at which individual cells exhibit a mechanosensitive response, often correlated with the stiffness of cells themselves [21]. Individual MDCK-II cells show mechanoresponse between 0.6 and $5 \mathrm{kPa}$, while no significant differences were noted on 11,20 , and $34 \mathrm{kPa}$ [34]. This result coincides with the range reported for in vivo stiffness of kidneys $[66,67]$ and stiffness of MDCKII cells themselves [68]. This range also coincides with the range at which we observe major changes in tissue organization and mechanoresponse.

\section{DISCUSSION}

Besides shedding new light on the emergence and the properties of the homeostatic state, the results obtained herein can be used to reconcile some of the apparently contradicting results discussed in the literature. Namely, the mechanoresponse in tissues is truly a cooperative effect involving a significant number of cells. Consequently, clusters containing up to three cells [29] are presumably dominated by the mechanoresponse of individual cells and not representative of the response of the ensemble. Clusters containing three to seven cells, in which no effect of substrate elasticity was found [29], are potentially too small and the 
fluctuations are too large to clearly capture changes in cell morphology. Nonetheless, even at these ensemble sizes, the biochemical response clearly couples to the mechanoresponse. For example, treatment of small MDCK-II clusters with a TGF- $\beta 1$ growth factor induced apoptosis on soft substrates $(<1 \mathrm{kPa})$, while on hard substrates $(>5 \mathrm{kPa})$, it led to the epithelial-mesenchymal transition [33].

Similar effects of cluster size were identified in fibroblasts and in epithelial cells seeded on hard gels and glass substrates. In small aggregates, effects of substrate elasticity were reminiscent of single-cell results [30], although under the same conditions, epithelial cell sheets showed no appreciable morphological response [31]. The same behavior has been reported for bovine aortic endothelial cells in confluent layers [32]. Notably, in those conditions, our own samples have shown a large variability. Actually, prior to homeostasis, changes in the distributions of morphological parameters within a single colony are significantly larger than the differences between distributions emerging from different homeostatic states. The exception is the homeostatic state observed on very soft gels, due to the very different growth patterns that are characteristic of growth below the nonequilibrium phase transition $[34,35]$. Last but not least, we note that if our colony meets a mechanical or chemically imposed edge, the homeostatic state will expand over the entire surface. The other compartments are thus dynamic, mechanoresponsive structures associated with development of the tissue. Consequently, the same tissues grown in systems of a few hundred nanometers in size do not show the $\mathrm{H}_{\mathrm{P}}$ compartment $[61,69]$. Nevertheless, cell density can reach values up to $10^{4}$ cells $/ \mathrm{mm}^{2}$, showing a mechanoresponsive behavior, where the $\mathrm{H}_{\mathrm{PL}}$ state is accurately recovered.

Strong fluctuations prevail even in the homeostatic state, as evidenced by the error bars in Figs. 1(c) and 2(b). This result demonstrates that mechanosensitivity of tissues must be investigated statistically. However, on hard gels (3-30 kPa), despite the averaging over homeostatic states occupying tens of square millimeters, we were not able to observe appreciable changes in the colony shape, confirming previous reports [31]. Nonetheless, the manipulation of cohesive and adhesive forces within MDCK-II cell clusters induces changes in the cell spreading areas and proliferation rates, showing that these systems are still sensitive to the cooperative generation of stress [36]. Nevertheless, significant differences between samples grown on hard gels and glass are observed only when a 2-mm-thick $\mathrm{H}_{\mathrm{P}}$ on gels as well as 0.5 -mm-thick $C_{2}$ and $C_{3}$ compartments on glass fully form about 4 days after seeding, which again points to the necessity for large sample sizes in studying cooperative behavior of cells in the tissue and the associated steady states.

\section{CONCLUSION}

To summarize, we clearly demonstrate that tissue homeostasis depends on the mechanical properties (i.e., Young's elastic modulus $E$ ) of the environment on multiple length scales. The observed self-organization produces several nonequilibrium phases, including a motile, nonproliferating compartment that is of higher density than the homeostatic state. The homeostatic state itself is found to undergo a nonequilibrium phase transition from columnar to squamous tissue by increasing the stiffness of the underlying matrix, as theorized previously [14]. Surprisingly, while the 3D cell shapes and densities change drastically during homeostasis on different matrices, the topology of the steady state is preserved, suggesting that homeostasis can be associated with a set of mechanically universal states.

It remains to be understood, however, what the developmental advantages of this particular state are. It is also not clear which cellular processes and signaling are involved in its maintenance. The intimate relation between the nuclear distributions and the tissue organization, as seen through the applicability of the set-based Voronoi tessellation, suggests that a cooperative shape optimization [70] may be involved. Yet, the physical forces and the biological signaling leading to optimization to these particular distributions need to be analyzed, the result of which may be associated with and relevant to tissue development where mechanosensitivity was already found to be important $[22,23]$. The mechanical universality of the homeostatic state should furthermore be verified in vivo where mechanical modulation of the environment occurs over long time periods. Tissues should be able to accommodate for these changes by actively maintaining their topology, and not the cell area, as well as the proliferation and apoptosis rate, a fact that should be further explored in a more physiological setting.

\section{ACKNOWLEDGMENTS}

This work was, in part, supported by the Deutsche Forschungsgemeinschaft (DFG) through the collaborative research center SFB 755 "Nanoscale Photonic Imaging," project B8 (F. R., C. W.), by the Deutsche Forschungsgemeinschaft (DFG)—SFB TRR 305 "Striking a moving target: From mechanisms of metastatic colonization to novel systemic therapies"- B05 (D. D.). A.S.S. and M.H. acknowledge fundings by the DFG "Mechanobiology in Epithelial 3D Tissue Constructs"363055819. Furthermore, we acknowledge DFG Research Training Group 1962 "Dynamic Interactions at Biological Membranes-from Single Molecules to Tissue" (A. S. S., S. G., and D. D.), the ERC StG 337 MembranesAct (A. S. S., S. K., J. L., and L. N.), intramural funds by the IZKF, Project No. A80 (D. D., S. G., and D. V.), and by the Emerging Fields Initiative "BigThera," which was partly supported by the Staedtler Foundation (A. S. S., D. D., and D. V.). We thank the Optical Imaging Center Erlangen (OICE) for their support.

A. S. S. conceived the study, and designed the experiments with F. R., the analysis tools with S. K., and the simulations with M.H.. A. S. S., F. R., and D. D. supervised the work. 
S. K., C. W., D. V., and S.G. performed the experiments. S. K., S. G., and M. H. performed structural analysis of the data using tools developed by S. K.. J. L. performed the analysis of topological measures using tools developed together with S. K. M. H. performed the simulations using the code developed by L. N.. D. V. performed the experiments and the analysis associated with velocity distributions. A. S. S. and M. H. wrote the paper with the help of F. R. and S. K.. Critical insight was provided by all authors.

\section{APPENDIX A: METHODS}

\section{Cell culture and gels preparation}

MDCK-II cells were obtained from ECACC, UK (Cat \#00062107, RRID:CVCL_0424) and cultured in MEM Earle's medium (\#F0325, Biochrom) supplemented with $5 \%$ fetal bovine serum (FBS, \#F0804, Sigma-Aldrich), $2 \mathrm{mM}$ L-glutamine (\#G7513, Sigma-Aldrich), and 1\% penicillin and streptomycin (\#15070-063, Gibco, LifeTechnologies) at $37^{\circ} \mathrm{C}$ and $5 \% \mathrm{CO}_{2}$. Cells were passaged every two or three days before reaching $80 \%$ confluence.

Elastic PA gels were prepared as described earlier [34]. In brief, appropriate mixtures of acrylamide (40\% solution, BioRad) and bis-acrylamide (2\% solution, BioRad) were polymerized by addition of $0.1 \%(\mathrm{v} / \mathrm{v}) \mathrm{N}, \mathrm{N}, \mathrm{N}, \mathrm{N}$ tetramethylethylenediamine (TEMED) and 1\%(v/v) ammonium persulfate (APS) for 60 minutes at RT on plasma-cleaned glass coverslips (No. 1, 25-mm $\varnothing$, VWR) that were pretreated with 3-aminoproyltriethoxysilane (APTES, SigmaAldrich) for 15 minutes and incubated with a $0.5 \%$ solution of glutaraldehyde in PBS (Sigma Aldrich) for 30 minutes. For quality control, Young's modulus $E$ was measured macroscopically by a bulk controlled with a strain rheometer (MCR 501, Anton Paar) using a cone and plate geometry and microscopically by atomic force microscopy (MFP-3D, Asylum Research, Santa Barbara). Typical results of both techniques are given in Fig. 5. We found that the standard deviation of Young's modulus $E$ is usually below $10 \%$. For quality control reasons, we used the stock solutions stored no longer than 3 months at $4{ }^{\circ} \mathrm{C}$ and protected from light and ideally prepared as many samples from the same batch as needed.

After polymerization, the PA gels were washed extensively with PBS and subsequently coated with Collagen-I (BD Biosciences) at $0.02 \mathrm{mg} / \mathrm{mL}$ in a $50 \mathrm{mM}$ HEPES buffer using the bifunctional cross-linker Sulfo-SANPAH (Pierce, Thermo Scientific) activated for 10 minutes with UV light $(365 \mathrm{~nm})$. Its homogeneity was tested and demonstrated on numerous occasions in Refs. [64,71,72]. In the current work, we use the conditions optimized in Ref. [64] [see Fig. 2(c) inset therein], at which the concentration of collagen on the surface is fully saturated, to avoid deviations in available collagen density, and to make sure that adhesive properties of the surfaces are identical in all cases.

One series of experiments involved all gels $(0.6,3,5,11$, and $21 \mathrm{kPa}$ ) prepared concurrently. For quality control of the PA gel, stock solution stiffness was measured regularly with a bulk rheometer [e.g., see Fig. 5(a)] as well as by AFM on the ready-made PA gels on coverslips [see Fig. 5(b)]. Several bottles of collagen-I were bought from the same batch. All MDCK-II cells came from the same passage and were pooled before seeding to ensure a homogeneous population. All colonies were seeded at once, cultured together under identical conditions, and treated in the same way during the experiment.

\section{Characterization of cellular features: Fluorescent staining and microscopy}

We characterize the cells constituting the mechanoresponsive tissues by imaging the following structures and features:

(i) Focal adhesions, which are macromolecular protein complexes located at the basal side of the cell. The main function is the reinforcement of the anchoring of the cells via integrins to ECM proteins. Besides mechanical linkage, it is involved in mechanosensing of the environment and the transduction of the
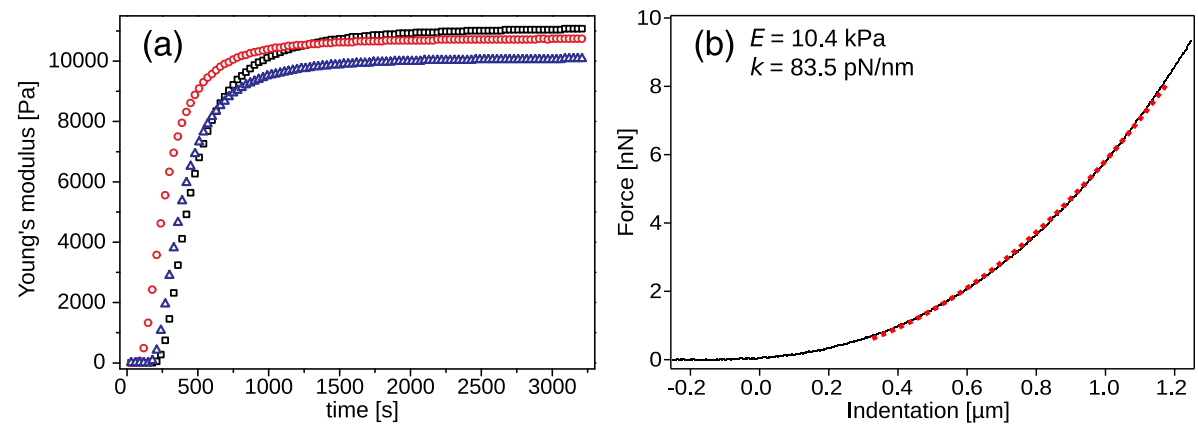

\begin{tabular}{|l|c|c|}
\multicolumn{1}{c|}{} & \multicolumn{2}{c|}{ AFM Measurement } \\
\cline { 2 - 3 } \multicolumn{1}{c|}{} & Mean [kPa] & St.dev. [kPa] \\
\hline Location 1 & 10.37 & 0.03 \\
Location 2 & 10.33 & 0.03 \\
Location 3 & 10.49 & 0.04 \\
Location 4 & 10.37 & 0.04 \\
Location 5 & 10.43 & 0.03 \\
Location 6 & 10.47 & 0.04 \\
Location 7 & 10.44 & 0.03 \\
Location 8 & 10.33 & 0.05 \\
Location 9 & 10.36 & 0.04 \\
\hline
\end{tabular}

FIG. 5. Characterization of the gel stiffness. (a) Bulk rheology measurement of Young's elastic modulus $E$ over time. Plateau values are $11.1 \mathrm{kPa}$ (black squares), $10.7 \mathrm{kPa}$ (red circles), and $10.1 \mathrm{kPa}$ (blue triangles), which yields an average value of $E=10.6 \pm 0.3 \mathrm{kPa}$. (b) AFM measurement of the force distance curve (black dots) and the fitted Hertz model (red dashed line) giving Young's modulus $E$ of the gels as $10.4 \mathrm{kPa}$. The table shows the mean values of the fitted Young's moduli $E$ of ten measurements at each of the nine locations on the PA gel. 
signal to the inside of the cell. When binding to ECM proteins (such as collagen, fibronectin, etc.), focal adhesion comprise paxillin in the intracellular compartment. The latter is a small protein $(\approx 65 \mathrm{kDa})$. It acts as an adapter for the recruitment of many other proteins to the cell membrane during adhesion to ECM and mechanotransduction.

(ii) Actin cytoskeleton, which is the main scaffold of the cell responsible for contractile stresses. It is built from actin and actin binding proteins (e.g., crosslinkers), which are proteins organized in filaments, bundles, and networks. Actin filaments are mostly located at the cell cortex and form together with myosin II mini-filament stress fibers at the basal and apical sides of the cells, the "cellular muscles," which exhibit contractile forces. Ventral stress fibers (at the basal side of the cells) are connected on both ends to focal adhesions.

(iii) Dividing cells using 5-ethynyl-2-deoxyuridine (EdU), which is a thymidine analogue that can be incorporated into DNA during its replication. EdU is an indicator that cells underwent the S-phase of the cell cycle.

For imaging, tissues were fixed using a $10 \%$ solution of formaldehyde (\#47608, Sigma-Aldrich) in PBS for 5 minutes, and the cells were permeabilised for 10 minutes using a $0.5 \%$ solution of Triton X 100 (Carl Roth). After washing with PBS, samples were blocked using 3\% BSA (\#A9418, Sigma-Aldrich) in PBS for 30 minutes at RT, which preceded another Triton X 100 treatment of 5 minutes at RT, followed by 3 washing steps with PBS.

Filamentous actin was stained using Phalloidin Atto 550 (\#AD550-81, Atto Tec) by incubating with a solution [1:250] in $3 \%$ BSA in PBS for $1.5 \mathrm{~h}$ at RT. Next, $\beta$-catenin was stained by incubating with the primary antibody (mouse, Sigma-Aldrich Cat \#C7082, RRID:AB_258995) solution [1:200] in 3\% BSA in PBS, for $2 \mathrm{~h}$ at RT. This was followed by the secondary antimouse $\operatorname{IgG}$ Alexa Fluor 488 (goat, \#SAB4600388, Sigma-Aldrich) [1:250] in 3\% BSA in PBS for 30 minutes at RT. Last, nuclei were stained using Hoechst 33342 (H3570, Invitrogen/Thermo Fisher Scientific) [1:1000] in 3\% BSA in PBS for 30 minutes at RT. EdU staining was performed using the Click-it Plus EdU AlexaFluor 488 Imaging Kit (\#C10637, Molecular Probes, Thermo Fisher Scientific) according to manufacturer's instructions. Cells were incubated with $10 \mu \mathrm{M}$ EdU for $4 \mathrm{~h}$. Paxillin staining was performed with a primary antibody antipaxillin (rabbit, Sigma-Aldrich Cat \#SAB4300384, RRID: AB_10626474) [1:1000] in 3\% BSA in PBS for $2 \mathrm{~h}$ at RT, followed by the secondary antibody antirabbit IgG Alexa Fluor 488 (goat, \#AP132JA4, Sigma-Aldrich) [1:250] in 3\% BSA in PBS for 30 minutes at RT. Samples were mounted using Fluoroshield histology mounting medium (\#F6182, Sigma-Aldrich, USA) on cover glasses $(26 \times 76 \mathrm{~mm}$, "Menzel Glaeser", \#1, Thermo Fisher Scientific).
Epifluorescence microscopy images were acquired on an inverted microscope (Zeiss Cell Obsever Z1) using $5 \times$ and N-Achroplan 20× objectives, using the AxioCam M3 and AxioVision software packages (all Zeiss). Confocal microscopy was performed on a Leica LSM SP5 laser-scanning microscope equipped with a white light laser and a $63 \times$ and a $100 \times$ oil immersion objective, yielding fields of view of $(246 \mu \mathrm{m} \times 245 \mu \mathrm{m})$ and $100 \times(155 \mu \mathrm{m} \times 155 \mu \mathrm{m})$, respectively. The step size in the $z$ direction was kept constant at $0.25 \mu \mathrm{m}$.

All tissues grown in one series were fixed and stained simultaneously and imaged consecutively without changing the microscope settings.

\section{APPENDIX B: HOMEOSTATIC STATE: IMAGING}

\section{Contact inhibition of proliferation and locomotion in homeostatic states}

To demonstrate the contact inhibition of proliferation, we search for cell divisions using EdU staining of the $\mathrm{H}_{\mathrm{PL}}$ state

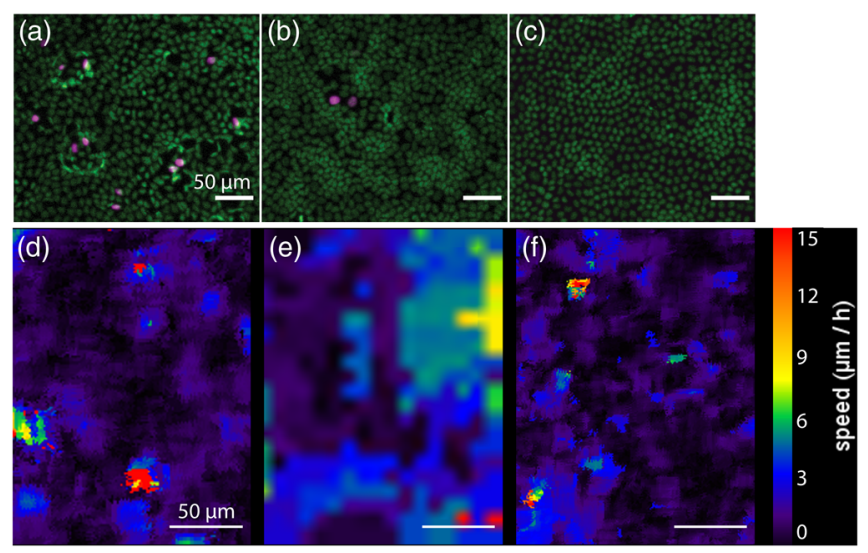

FIG. 6. Contact inhibition with respect to proliferation and motion. Illustration of the contact inhibition for proliferation using EdU staining (top row) and for motion through PIV analysis (bottom row). (a)-(c) Images of EDU (purple) and nuclei (in green) stained $\mathrm{H}_{\mathrm{PL}}$ for glass, hard gels, and soft gels, respectively, showing no cell divisions in the $\mathrm{H}_{\mathrm{PL}}$. Both on glass and on gels, the majority of the division events were observed in the vicinity of a defect in a tissue. The scale bar is $50 \mu \mathrm{m}$. (d)-(f) PIV velocity fields within the $\mathrm{H}_{\mathrm{PL}}$, showing low cell mobility on soft gels, $30 \mathrm{kPa}$ gels, and glass, respectively. (d) MDCK-II cells seeded on a soft gel substrate with a Young's modulus of $0.75 \mathrm{kPa}$ in a $2 \mu \mathrm{L}$ dense droplet of 100000 cells on a covalently bound collagen-I coated surface, imaged 2.5 days after seeding, with a pixel size of $1.25 \mu \mathrm{m}$, and time steps of $15 \mathrm{~min}$. (e) MDCK-II cells seeded on a hard gel substrate with a Young's modulus of $30 \mathrm{kPa}$ in a $2 \mu \mathrm{L}$ dense droplet of 10000 cells on a covalently bound collagen-I coated surface, imaged 2.7 days after seeding, with a pixel size of $10 \mu \mathrm{m}$ and time steps of $20 \mathrm{~min}$. (f) MDCK-II cells seeded on a glass substrate in a $2 \mu \mathrm{L}$ dense droplet of 10000 cells on a collagen-I coated surface, imaged 10 days after seeding, with a pixel size of $0.886 \mu \mathrm{m}$ and time steps of $20 \mathrm{~min}$. 

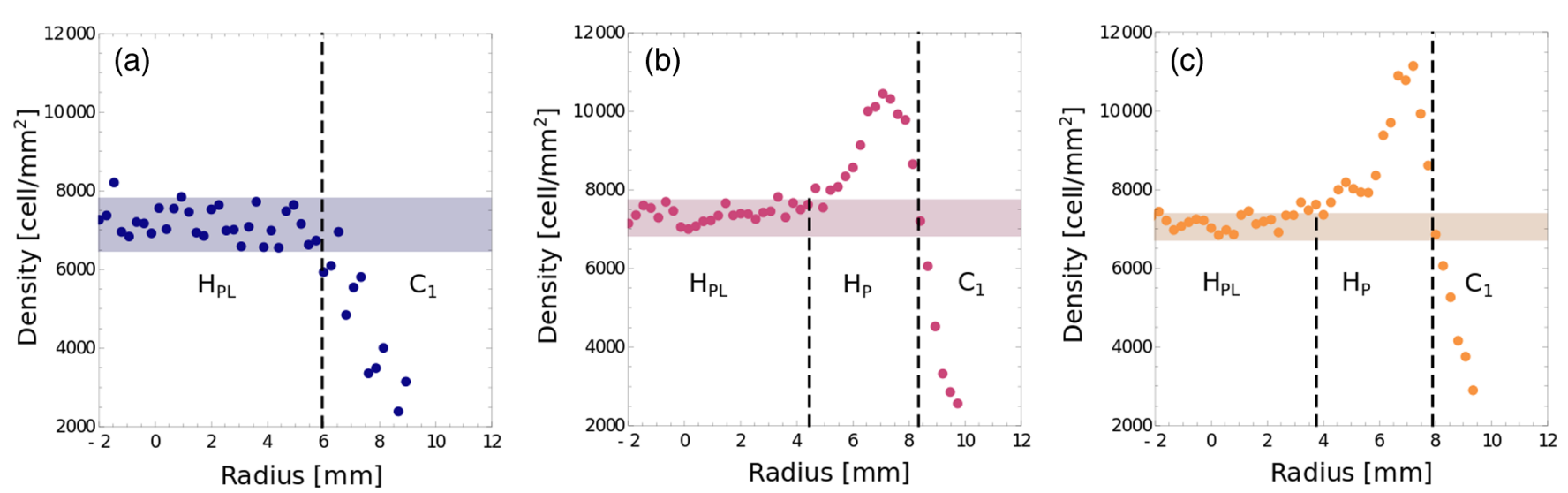

FIG. 7. Spatial dependence of the density in the cluster. (a) Cluster on the glass substrate, (b) cluster on $11 \mathrm{kPa}$ PA gels, and (c) cluster on $5 \mathrm{kPa}$ gels, showing very similar features to the cluster grown on $11 \mathrm{kPa}$ gels. These colonies belong to two different series of data, in comparison to the ones shown in Fig. 1, but are mutually consistent. Horizontal lines represent 2 standard deviations around the mean cell density in the central region (2.3 mm radius). If the image segment is within those lines, it is considered as bulk. If the density of a given image segment is below the bottom line, the segment is considered as part of the edge region, and segments with density higher than the upper line are considered as part of the high-density ring.

on glass, and hard and soft gels [see Figs. 6(a)-6(c)] following the above-described protocol. To demonstrate the inhibition of locomotion, PIV analysis (vide infra) was performed, and it showed only slow positional fluctuations of cells [see Figs. 6(d)-6(f)].

\section{Density in the homeostatic state on glass and hard gels}

On glass and hard gels, we first evaluate the cell density $\rho_{0}$ in the central region of the clusters $(2.3-\mathrm{mm}$ radius around the cluster center, tiled into $267 \mu \mathrm{m} \times 575.6 \mu \mathrm{m}$ fields of view). The standard deviation $\sigma_{p}$ is evaluated as the deviation in cell density obtained from different fields of view. Hence, $\sigma_{p}$ is a measure of fluctuation densities in the sample. The homeostatic state is defined as all fields of view showing a density in the range of $\rho_{0} \pm 2 \sigma_{p}$. The $\mathrm{H}_{\mathrm{P}}$

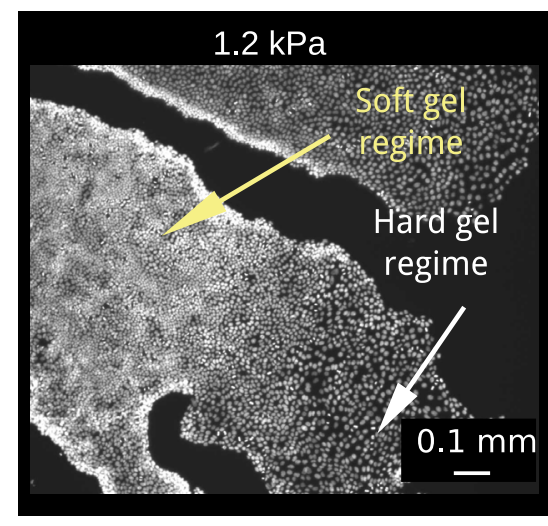

FIG. 8. Tissues grown on soft gels with stiffness of $1.2 \mathrm{kPa}$. For slightly higher stiffness than $0.6 \mathrm{kPa}$, typically in the range of $1 \mathrm{kPa}$ to $3 \mathrm{kPa}$, the tissue exhibits the behaviors expected on both soft gels and hard gels, as indicated on the picture by arrows. The image is from Ref. [73]. state on gels is determined as all fields of view in which the density is higher than $\rho_{0} \pm 2 \sigma_{p}$ (see Fig. 7). The average cell area is calculated from the mean cell density. The latter was obtained from hand-corrected segmented images of cell nuclei (accuracy above 99\%) and estimated using a MATLAB routine discussed in detail in our previous publication [34].

With regards to the density in the range $E \in[3: 21] \mathrm{kPa}$, the average densities for the homeostatic states $\rho\left(\mathrm{H}_{\mathrm{PL}}\right)$ and $\rho\left(\mathrm{H}_{\mathrm{P}}\right)$ (when relevant) are reported in Table I.

When reporting the density in the $\mathrm{H}_{\mathrm{P}}$ state in Fig. 1, the averages and the standard deviations are found from the entries in Table I. It is notable that this variance is very similar to $\sigma_{p}$ : It is somewhat larger on glass than on hard gels, and it is the largest on soft gels. With these data (comprising more than 850000 cells), we cannot establish statistically different results for average densities of the $\rho\left(\mathrm{H}_{\mathrm{PL}}\right)$ and the $\rho\left(\mathrm{H}_{\mathrm{P}}\right)$ on hard gels.

\section{Density of colonies grown on soft gels}

Following the previously established criteria (see Ref. [34]), colonies smaller than $2.8 \times 10^{-2} \mathrm{~mm}^{2}$ are classified as small. Below this size, we presume that finite-size effects still play a role in the monolayered structure. For the analysis of the density in small clusters, we use the entire monolayer. In the analysis of the large cluster density, averaging was performed over a significant section of the monolayer for which there is no other compartment in the same field of view. The same criteria were applied in determining the topology-related measures, which were extracted only for large clusters (size $>2.8 \times 10^{-2} \mathrm{~mm}^{2}$ ). The scars left by extrusions (see Appendix F) are removed manually from the images since cells cannot be counted in these areas. Here, scars left from the merging of colonies have very little effect, if any. 
TABLE I. Information about the amount of cells considered in obtaining the density of the homeostatic states.

\begin{tabular}{lcccc}
\hline \hline Substrate & $\rho\left(H_{P L}\right)\left(\right.$ cells $\left./ \mathrm{mm}^{2}\right)$ & Number of cells & $\rho\left(H_{P}\right)\left(\right.$ cells $\left./ \mathrm{mm}^{2}\right)$ & Number of cells \\
\hline $0.6 \mathrm{kPa}$ & $12.58 \times 10^{3}$ & $500 \times 10^{3}$ & & \\
$3 \mathrm{kPa}$ & $7.71 \times 10^{3}$ & $26 \times 10^{3}$ & $8.31 \times 10^{3}$ & $23 \times 10^{3}$ \\
$5 \mathrm{kPa}$ & $7.12 \times 10^{3}$ & $34 \times 10^{3}$ & $7.56 \times 10^{3}$ & $5 \times 10^{3}$ \\
$5 \mathrm{kPa}$ & $7.08 \times 10^{3}$ & $45 \times 10^{3}$ & $8.49 \times 10^{3}$ & $31 \times 10^{3}$ \\
$11 \mathrm{kPa}$ & $7.37 \times 10^{3}$ & $56 \times 10^{3}$ & $8.81 \times 10^{3}$ & $26 \times 10^{3}$ \\
$21 \mathrm{kPa}$ & $7.14 \times 10^{3}$ & $57 \times 10^{3}$ & $8.00 \times 10^{3}$ & $14 \times 10^{3}$ \\
Glass & $6.45 \times 10^{3}$ & $10 \times 10^{3}$ & & \\
Glass & $7.11 \times 10^{3}$ & $9 \times 10^{3}$ & & \\
Glass & $7.02 \times 10^{3}$ & $8 \times 10^{3}$ & & \\
\hline \hline
\end{tabular}

\section{Cell volume measurements}

Samples of cell monolayers grown on $0.6 \mathrm{kPa}, 5 \mathrm{kPa}$, and glass substrates were stained for actin and imaged with confocal microscopy (see details in Appendix A). The cell height was estimated 4 days after seeding to make sure there was a small amount of defects within the monolayers. The density of the tissue segments is calculated by counting cells. The following values have been obtained: on glass, 6546 cells $/ \mathrm{mm}^{2}$; on $5 \mathrm{kPa}$ gels, 6944 cells $/ \mathrm{mm}^{2}$; in the large cluster on $0.6 \mathrm{kPa}$ gels, 14760 cells $/ \mathrm{mm}^{2}$; and in the small cluster on $0.6 \mathrm{kPa}$ gels, 23010 cells $/ \mathrm{mm}^{2}$. For hard gels and glass, the densities are somewhat smaller than the average reported for the $\mathrm{H}_{\mathrm{PL}}$ state, yet within 2 standard deviations of density fluctuations $\sigma_{p}$ used to define the state. In this way, we were able to balance the accuracy of two independent measurements-density and height.

The cell height is given by the mean intensity of the actin signal in the stacks. These stacks are plotted as a function of the $z$-axis position as shown in Fig. 1. The corresponding images have been taken at day 4 after seeding instead of day 6 to avoid any defects within the tissue that typically arise when the tissue ages. This approach also leads to slightly smaller densities than the one reported in Fig. 1(c), as indicated in the labels in Fig. 1(d). More information about the time evolution of the density in the homeostatic state can be found in Fig. 12. The sampled showed an actin profile with two maxima corresponding to the positions above and under the cell nuclei [see Fig. 1(d)]. Intensity at the beginning $\left(I_{\text {bottom }}\right)$ and the end $\left(I_{\text {up }}\right)$ of the cell monolayer is estimated via the two inflection points of the curves. To associate an error with this definition of the bottom and top of the cell, we take an error of $\pm 5 \%$ of the intensity at the inflection point divided by the gradient at the inflection point. This method therefore associates larger $z$-axis estimation errors to the slowly decaying actin intensity profiles. The cell area is estimated from the density at the homeostatic state on the corresponding gels.

The average cell volume is obtained as a product of the mean cell area and the mean cell height. The deviations of the volume are calculated by propagating the respective errors in the cell area and height.

\section{APPENDIX C: HOMEOSTATIC STATE: CHARACTERIZATION}

\section{Morphological characterization}

To examine the properties of the cells and their nuclei, we select, in each tissue, a number of regions of interest from the original $20 \times$-magnified images (with a pixel size of $0.31 \mu \mathrm{m}$ ), in order to avoid the effects of defects and domes on glass. All regions were collected in the homeostatic state, far enough from the external compartments. On glass, we sampled 35 regions of interest (areas between 14 000 and $78000 \mu \mathrm{m}^{2}$ ) providing 2575 cells for morphological analysis. On $5 \mathrm{kPa}$, in total 9095 cells were analyzed from 24 regions $\left(28000-128000 \mu \mathrm{m}^{2}\right)$. On $11 \mathrm{kPa}$ PA gels, we have 5244 cells from 27 regions (28000 and $128000 \mu \mathrm{m}^{2}$ ). All morphological measures were obtained using a previously described procedure $[34,40]$, from the SVT. Because of the balance of forces, this tessellation is particularly suitable for analyzing the homeostatic states, as the error in determining all morphological measures is between $5 \%$ and $8 \%$, depending on the cell size in comparison to pictures of $\beta$-catenin stained tissues. A more in-depth description of the precision of this method for the densities discussed in this paper can be found in Table II.

TABLE II. Quality of SVT-extracted measures. Relative average deviation of the SVT-based morphological measures from the measures obtained via $\beta$-catenin stained, segmented, and handcorrected pictures of the membrane, of fully tubular cells within the $\mathrm{H}_{\mathrm{PL}}$. As discussed in our previous work, these deviations are comparable to or smaller than deviations that are inherent to uncorrected $\beta$-catenin segmented images [38].

\begin{tabular}{lcccc}
\hline \hline Substrates & Area & Perimeter & Elongation & Neighborhood \\
\hline Soft gels & $6.62 \%$ & $4.20 \%$ & $6.90 \%$ & $3.77 \%$ \\
Hard gels & $6.86 \%$ & $5.10 \%$ & $8.97 \%$ & $6.64 \%$ \\
Glass & $10.54 \%$ & $5.52 \%$ & $10.27 \%$ & $6.92 \%$ \\
\hline \hline
\end{tabular}


(a) $\quad 13000$ cells $/ \mathrm{mm}^{2}$

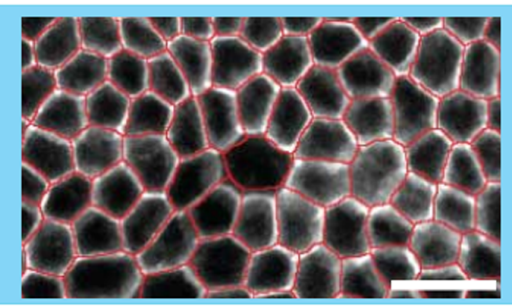

(b)
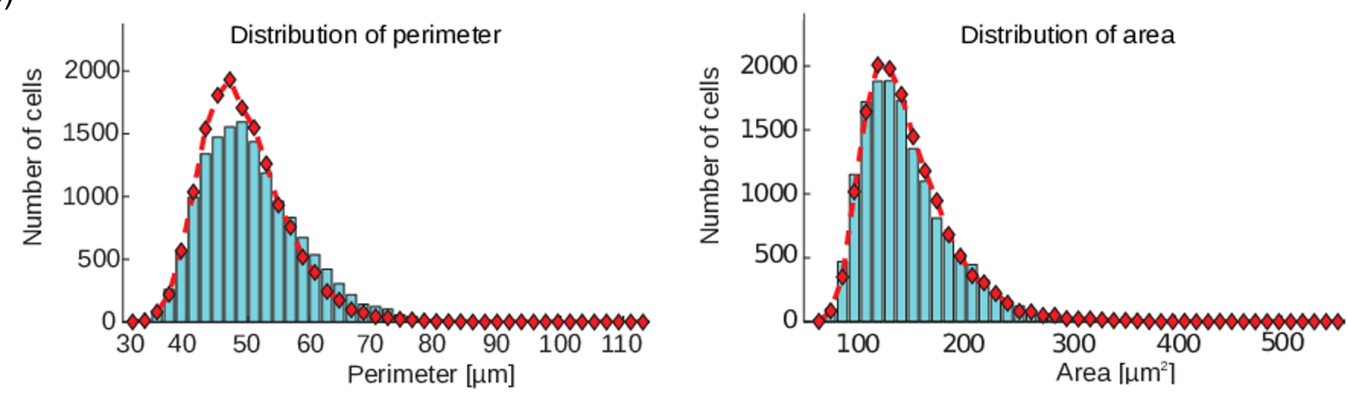

FIG. 9. Morphological analysis of the tissue. (a) Comparison between pictures of the cell membranes stained with $\beta$-catenin and the set-based Voronoi tessellation (in red). (b) Distribution of cell perimeters and areas, respectively, measured by pictures of $\beta$-catenin stained membranes (bars) and by SVT (red diamonds) for cells grown on hard $11 \mathrm{kPa}$ gels. These distributions comprise cells from all compartments of the tissue, including the edge compartments. Prior to the comparison, all segmented images of $\beta$-catenin and nuclei have been hand corrected for errors, as described in Refs. [38,73].
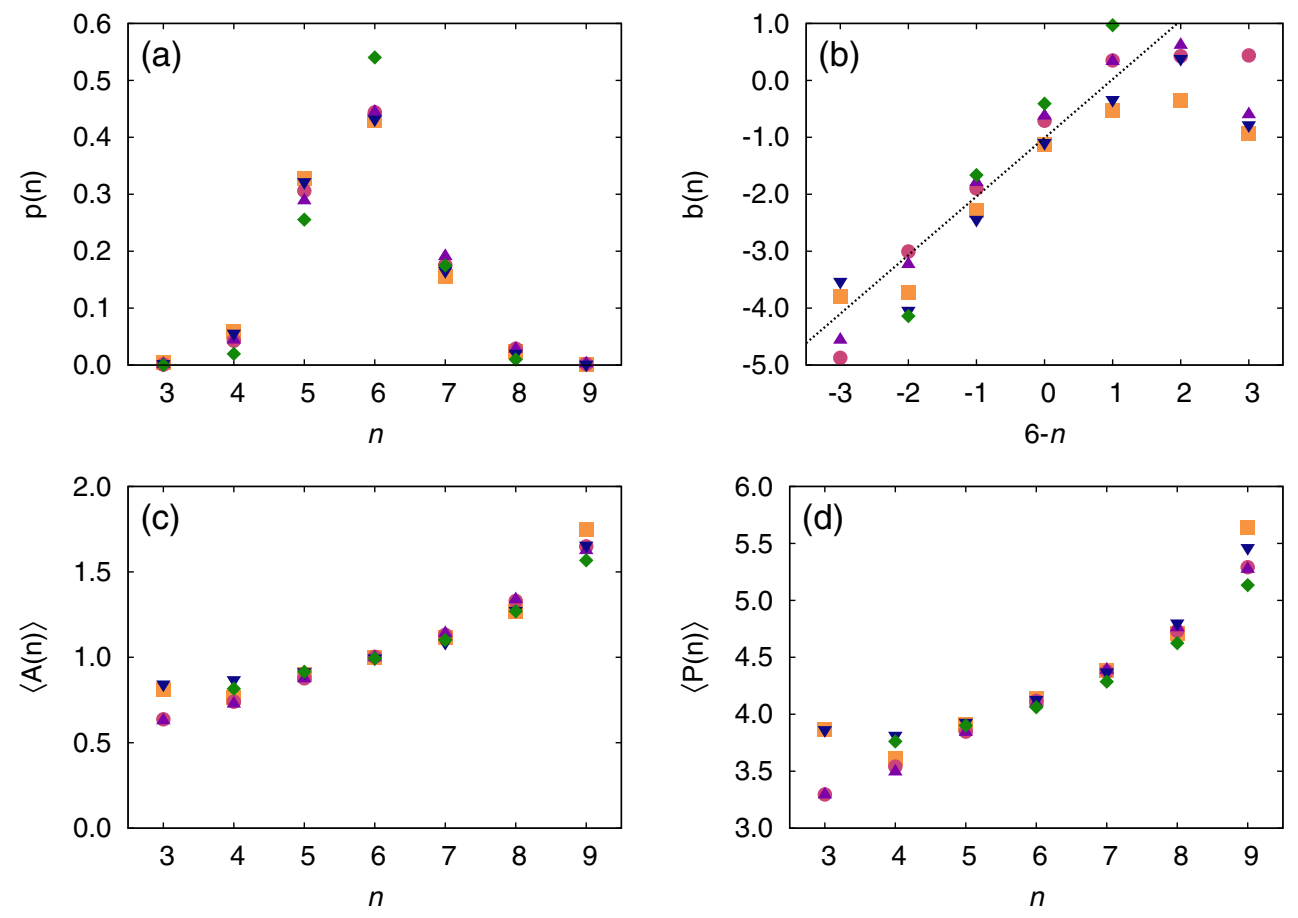

FIG. 10. Topological equivalence of tissues on different substrates. (a) Histogram of the number of neighbors per cell for each substrate. (b) Aboav-Weaire's law for each substrate. The black line seen on the figure is a fit on the linear part of the data. (c) Lewis' law for each substrate. (d) Desh's law for each substrate. The graphs consider tissues grown on all substrates: $\mathrm{H}_{\mathrm{PL}}$ glass (blue down-triangle), $\mathrm{H}_{\mathrm{PL}} 11 \mathrm{kPa}$ gels (purple up-triangle), $\mathrm{H}_{\mathrm{P}} 11 \mathrm{kPa}$ gels (green diamonds), $\mathrm{H}_{\mathrm{PL}} 5 \mathrm{kPa}$ gels (pink circle), and $\mathrm{H}_{\mathrm{PL}} 0.6 \mathrm{kPa}$ gels (orange squares). For the last two laws, the area $A$ of each cell and its perimeter $P$ have been divided by the cell mean area and its square root, respectively. 
Pictures illustrating the quality of the tessellation with respect to the $\beta$-catenin stained pictures can be found in Fig. 9.

Error bars in Fig. 2(b) have been obtained by considering the distributions of 50 statistically independent subsets of cells. For $n$ cells considered on a given substrate, statistically independent subsets of $n / 2$ cells were considered. The distributions of cell area and perimeters, nucleus area and perimeters, and nuclei and cell elongation have been computed for those 50 subsets, always using the same binning. For each bin, the average value and standard deviation of those 50 iterations were computed. The error bars displayed in Fig. 2(b) are centered around the average value, and the size of the bars is given by \pm the standard deviation of all 50 average values.

\section{Topological characterization}

To describe the topological properties of tissues grown on substrates of different stiffnesses, we focus on three different geometric laws. The first law, Aboav-Weaire's law, linearly correlates the number of neighbors of a cell with $n$ sides and the average number of neighbors $m(n)$ that cells adjacent to ones with $n$ sides have [41-43]. Mathematically, it can be written as

$$
\langle m(n)\rangle=6-\gamma+\left(6 \gamma+\sigma_{n}^{2}\right) / n,
$$

where $\sigma_{n}^{2}$ is the second central moment of the distribution of $n$, and $\gamma$ is a constant. The two other laws describe the linear relationship between the number of neighbors $n$ and the average area $\langle A(n)\rangle$ of a cell with $n$ neighbors:

$$
\langle A(n)\rangle=\mu_{A}(1+\alpha(n-6)) .
$$

Here, $\mu_{A}$ is the average area of the cell in the assembly, and it is a constant. A similar expression, namely,

$$
\langle P(n)\rangle=\mu_{P}(1+\beta(n-6)),
$$

exists for the average perimeter $\langle P(n)\rangle$ of cells with $n$ neighbors and is known as Desh's law [53].

These laws are illustrated in Fig. 10, for the $\mathrm{H}_{\mathrm{PL}}$ tissues on all substrates, and also for the $\mathrm{H}_{\mathrm{P}}$ state observed on hard $11 \mathrm{kPa}$ gels.

\section{APPENDIX D: ANALYSIS OF THE COLONY STRUCTURE}

\section{Density profiles from high resolution imaging}

On glass and hard gels, after seeding a droplet, a confluent monolayer is formed 12 hours after seeding. Consequently, small groups of cells may exist only in the very early stages of the experiment and are a likely consequence of the initial seeding. As the colony grows

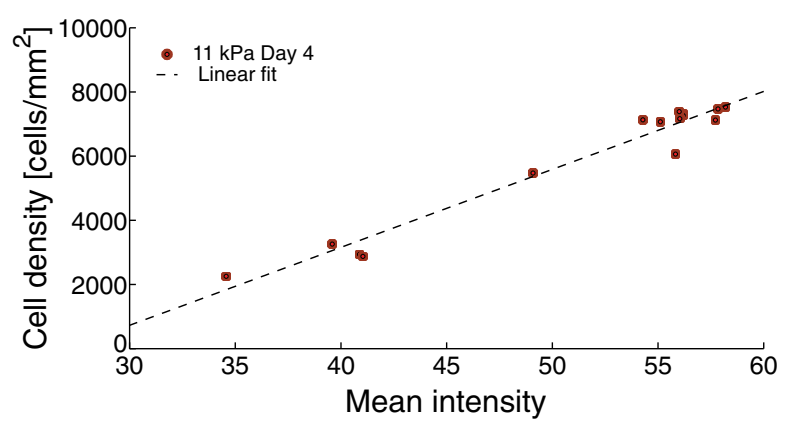

FIG. 11. Relation between cell number density and mean intensity of the confocal images. During image post-treatment, cell clusters are divided into equidistant rings, where the central region is a circle with a radius of $350 \mu \mathrm{m}$. The image segments taken from the different regions in the cell clusters are analyzed, and a linear mapping between average cell density and image intensity is found for each cluster. The figure illustrates the linear mapping extracted from the experiments realized on day 4 for tissues grown on $11 \mathrm{kPa}$ gels.

beyond the edges of the initial seeded drop, no secondary colonies are observed alongside the main one.

The cell density profile throughout the cell colony is analyzed after fixing the samples 4 or 6 days after seeding. High-precision measurements are obtained from images of cell nuclei, imaged at a magnification of $20 \times$. All images were segmented and hand corrected to achieve a fidelity of 99\%, as described in detail in previous work [38].

In fully reconstructed clusters, imaged at $5 \times$ magnification, density profiles are extracted from the calibrated fluorescence intensities. The grids of images were stitched using the $\mathrm{Fiji}^{3}$ plugin based on the work published by Preibisch et al. [74]. Cell density distributions were estimated from the stitched images of the Hoechst stained cell nuclei. First, the mean intensity was determined in $51-\mu \mathrm{m}$-wide circular rings drawn around the geometric center of the cluster (see Fig. 11). Consequently, linear mapping of the mean intensity to the mean cell density was performed for each tissue individually, after benchmarking based on at least 20 different segments with an area of at least $0.5 \mathrm{~mm}^{2}$, where the cell number and fluorescence intensity could be evaluated with very high accuracy.

\section{Dynamical analysis of the homeostatic states}

Locomotion within the $\mathrm{H}_{\mathrm{P}}$ state is demonstrated by imaging its position relative to the center of the tissue on day 4 and day 6 as shown in Fig. 12.

\section{PIV analysis and speed profiles}

To determine velocity profiles, tissues were grown for 6 days on glass. For this picture, 10000 MDCK-II cells were seeded in a dense droplet into a collagen-I coated well of an ibidi 2-well glass chamber slide. Six days after seeding, cell imaging was performed on a Zeiss AXIO 


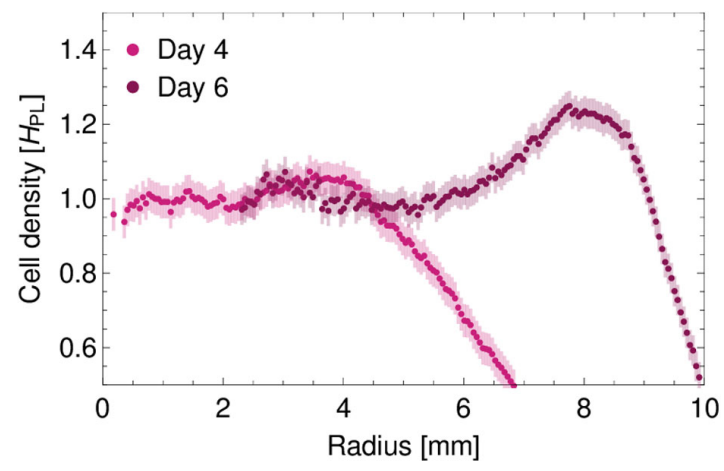

FIG. 12. Mobility in the $\mathrm{H}_{\mathrm{P}}$ compartment. Evolution of the density profile as a function of time for a tissue grown on hard gels, at day 4 (light pink) and at day 6 (dark pink). The weak local maximum at day 4 is seen to move from $4 \mathrm{~mm}$ to the center to $8 \mathrm{~mm}$ to the center at day 6 , evidencing the motion of the cell within the $\mathrm{H}_{\mathrm{P}}$ state. Error bars correspond to a typical variation of \pm 300 cells on the measurement of the density in the segment. The data in the first $2 \mathrm{~mm}$ from day 6 were omitted for clarity since, at this distance, the density of the bulk compartment has been achieved.

Observer.Z1 microscope with a moving stage and an incubation chamber capable of long-term cell incubation. For fluorescent visualization, the cluster was stained with CellTrace Violet live cell dye (Invitrogen). Time steps were 15 minutes apart, and the duration of the complete time lapse was $6 \mathrm{~h}$. In total, 25 time points were sampled (24 time steps between them). The full image was stitched out of 9 fields of view $(3 \times 3$ and $5 \%$ overlap in the $X$ and $Y$ directions). Fields of view had individual sizes of $1.4 \mathrm{~mm} \times 1.4 \mathrm{~mm}$ and a pixel size of $2.76 \mu \mathrm{m}$.

After imaging, a PIV analysis was performed with an ImageJ plugin as described in Ref. [75]. PIV interrogation windows were squares of size $44 \mu \mathrm{m}$ (16 px), correlated with search windows in the subsequent time step of $88 \mu \mathrm{m}$ (32 px) size. Grid distance between interrogation windows was $22 \mu \mathrm{m}$ (8 px, 50\% overlap).

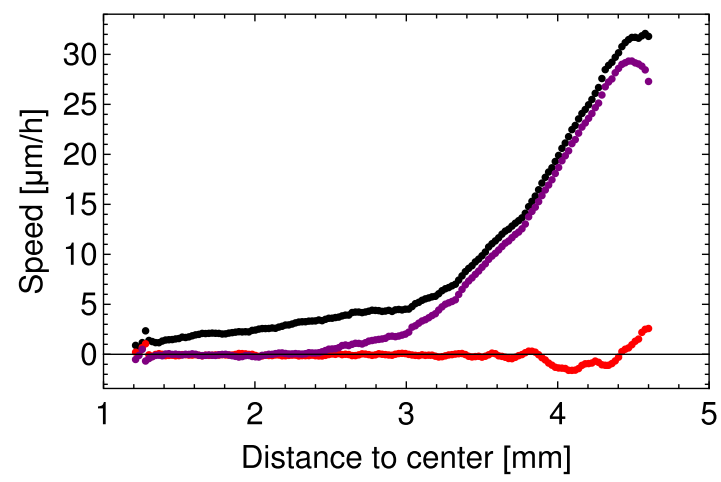

FIG. 13. Cell velocity and its components inside the tissue and saturation of the cell speed at the edge. Through PIV analysis (see Appendix D3), the cell speed (in black) and its components (radial in purple, tangential in red) are obtained.
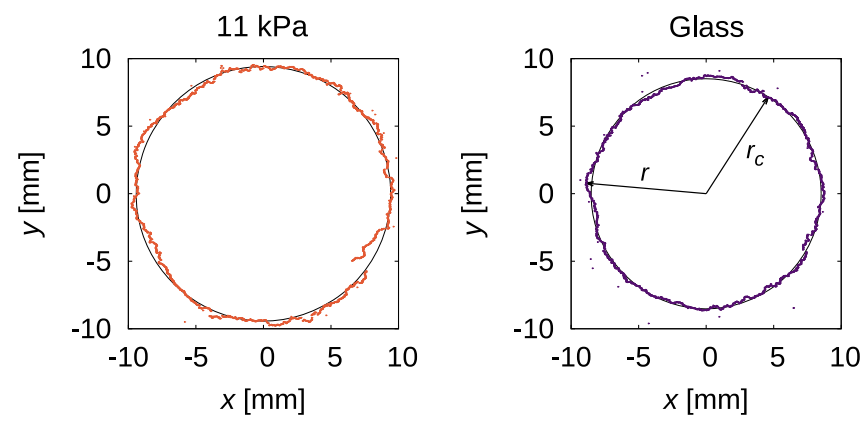

FIG. 14. Evaluation of the colony roughness. We show the edge of the cluster grown on $11 \mathrm{kPa}$ gels and glass, respectively, and the corresponding fitted circle to evaluate the roughness of the border. The vector $r$ corresponds to the distance of each pixel to the circle center, and $r_{c}$ is the circle center.

Visualization of the PIV-calculated velocity field was achieved in post-processing. Within a separate subsection of the ImageJ PIV plugin, a color plot was created by using the originally calculated velocity text file as input. The colored pixel had its center positioned at the $(x, y)$ coordinates. Colors were depicted depending on the speed magnitude.

For the analysis of the homeostatic state (Fig. 6), PIV analysis was performed on a region of interest of a square of $0.4 \mathrm{~mm} \times 0.4 \mathrm{~mm}$, subdivided into PIV interrogation windows of $16 \times 16 \mathrm{px}^{2}$. The latter were correlated via search windows of $32 \times 32 \mathrm{px}^{2}$. The grid distance between interrogation windows was of $2 \mathrm{px}$, therefore leading to an overlap of $87.5 \%$. A total of 25 images taken at 15 minutes intervals were considered, giving 24 velocity matrices. The average of those 24 matrices gives the results in Fig. 13.

\section{Roughness of the colony edge}

To calculate the distribution of roughness, first the edge is determined using a homemade algorithm applied on the thresholded images. The algorithm finds the first nonzero intensity pixel on lines drawn from the center of the images to the pixels of the border of the images, which gives the coordinates of the pixels of the edge of the tissue. On the ensemble of coordinates of each pixel describing the edge, we fit a circle with variable center and radius, which well describes the average colony shape, as shown in Fig. 14. Consequently, the distance of each edge pixel to the center of the circle $r$ is determined, and the deviation from the circle radius $r_{c}$ is recorded in the histograms displayed in Fig. 3.

\section{APPENDIX E: SIMULATIONS}

The numerical model is adapted from previous works [56-58]. Each cell is made of two points, which are sources and targets of all forces in the system. The "nucleus" of each cell is taken as the geometrical center of these two points. Finally, cell shape and geometrical features are 
TABLE III. Parameters used in the simulation and expressed in simulation units (s.u.). The values of the parameters listed as "variable" change throughout the article.

\begin{tabular}{lccccc}
\hline \hline Parameter & Symbol & Value & Parameter & Symbol & Value \\
\hline Mass & $m$ & $5.76 \times 10^{-4}$ & Background dissipation & $\mu_{\mathrm{bg}}$ & 0.1 \\
Integration time step & $d t$ & $10^{-5}$ & Intracellular dissipation & $\mu_{\mathrm{int}}$ & 1.1 \\
Growth strength & $B$ & 4.0 & Parallel dissipation & $\mu_{\|}$ & 0.2 \\
Growth force offset & $r_{0}$ & 1.12 & Perpendicular dissipation & $\mu_{\perp}$ & 0.15 \\
Volume exclusion & $f_{0}$ & Variable & Range of dissipation forces & $R_{t}$ & 0.8 \\
Adhesion & $f_{1}$ & 0.30 & Lower division threshold & $R_{c, 1}$ & 0.75 \\
Range of cell interaction & $R_{p p}$ & 1.0 & Upper division threshold & $R_{c, 2}$ & 0.85 \\
Active force strength & $\Gamma$ & Variable & Offset at division & $r_{c}$ & $1.12 \times 10^{-5}$ \\
Fluctuations & $\beta$ & 0.424 & Cell death rate & $k_{d}$ & $10^{-3}$ \\
\hline \hline
\end{tabular}

obtained by Voronoi tessellation based on the position of all nuclei.

The forces applied to building particles are a growth force $\vec{F}_{g}$ in order to trigger cell division, a cell-cell interaction $\vec{F}_{c}$, an active motile force $\vec{F}_{a}$, and a random force for inherent fluctuations $\vec{F}_{r}$. The expressions for these forces are the following:

$$
\vec{F}_{g}(\vec{r})=\frac{B}{\left(\left|\vec{r}_{i j}\right|-r_{0}\right)^{2}} \frac{\vec{r}}{|\vec{r}|},
$$

where the force only applies between the building particles within a single cell, $B$ and $r_{0}$ are parameters, and $\vec{r}$ is the vector pointing from one building point to the other,

$\vec{F}_{c}(\vec{r})= \begin{cases}f_{0}\left(\frac{R_{p p}^{5}}{|\vec{r}|^{5}}-1\right) \frac{\vec{r}}{|\vec{r}|} & \text { if }|\vec{r}|<R_{p p} \\ -\frac{f_{1}}{|\vec{r}|}|\vec{r}| & \text { if } R_{p p}<|\vec{r}|<1.4 R_{p p} \\ 0 & \text { if } 1.4 R_{p p}<|\vec{r}|,\end{cases}$

where the force applies between building particles of different cells only; $f_{0}, f_{1}$, and $R_{p p}$ are parameters; and $\vec{r}$ is the vector pointing from one building point to the other,

$$
\vec{F}_{a}=\Gamma \vec{e}, \quad \vec{e}=\frac{\vec{v}}{|\vec{v}|},
$$

where $\Gamma$ is a parameter and $\vec{e}$ is the unit vector pointing in the direction of the cell nucleus speed, defined as the average speed of its two building particles,

$$
\vec{F}_{r}=\beta \vec{e}_{r},
$$

where $\beta$ is drawn from a normal distribution with zero mean and standard deviation $\sigma$, while $\vec{e}_{r}$ is a random unit vector with an orientation drawn from a uniform distribution. These two last forces are applied to all building points in the system.

Dissipation within the simulations is accounted for thanks to the following forces. For the dissipation with the background, each building particle is submitted to

$$
\vec{F}_{v}^{b g}=-\mu_{b g} \vec{v},
$$

where $\vec{v}$ is its velocity. Dissipation within a single cell is accounted for by the force

$$
\vec{F}_{v}^{\text {int }}=-\gamma_{\text {int }} \omega^{2}\left(\vec{v}_{i j} \cdot \frac{\vec{r}_{i j}}{\left|\vec{r}_{i j}\right|}\right) \frac{\vec{r}_{i j}}{\left|\vec{r}_{i j}\right|} .
$$

Dissipation in the relative motion of cells is decomposed into a direction parallel and perpendicular to the line joining the building particles of each cell involved. One has

$$
\vec{F}_{v}^{\|}=-\gamma_{\|} \omega^{2}\left(\vec{v}_{i j} \cdot \frac{\vec{r}_{i j}}{\left|\vec{r}_{i j}\right|}\right) \frac{\vec{r}_{i j}}{\left|\vec{r}_{i j}\right|},
$$

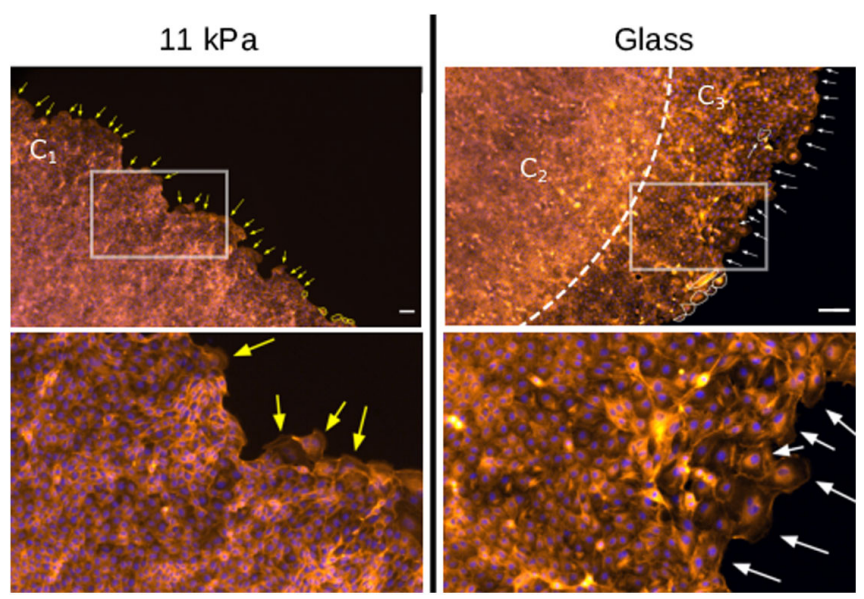

FIG. 15. Identifying leading cell phenotype. Leading cells are identified in tissues grown on hard gels (left) and glass (right). The red channel is used for actin, while the blue channel is for the nuclei. The arrows point to leader cells, and the scale bars are $100 \mu \mathrm{m}$. Leader cells are, on average, larger on glass substrates [white encirclement on glass (right), yellow encirclement on gels (left)]. In contrast to gels, leader cell phenotypes [55] on glass can sometimes be seen deeper inside the tissue and not only on the borderline. The bottom row corresponds to the zoomed-in images corresponding to the highlighted rectangles in the top row. 


$$
\vec{F}_{v}^{\perp}=-\gamma_{\perp} \omega^{2}\left(\vec{v}_{i j}-\left(\vec{v}_{i j} \cdot \frac{\vec{r}_{i j}}{\left|\vec{r}_{i j}\right|}\right) \frac{\vec{r}_{i j}}{\left|\vec{r}_{i j}\right|}\right) .
$$

The weight function $\omega$ appearing in the previous formula is written

$$
\omega= \begin{cases}1-\frac{\left|\vec{r}_{i j}\right|}{R_{t}} & \text { if }\left|\vec{r}_{i j}\right|<R_{t} \\ 0 & \text { otherwise. }\end{cases}
$$

The equations of motion for each building particle are

$$
m \overrightarrow{\ddot{r}}=\vec{F}_{c}+\vec{F}_{g}+\vec{F}_{a}+\vec{F}_{r}+\vec{F}_{v}^{\mathrm{bg}}+\vec{F}_{v}^{\mathrm{int}}+\vec{F}_{v}^{\|}+\vec{F}_{v}^{\perp} .
$$

These equations are integrated for each cell using a Euler algorithm with time step $d t$.

Cell division, as well as cell death, is included in the simulations. Cell death consists in the removal of a random cell at rate $k_{d} d t$ at each time step. Cell division is based on the distance between the two building particles $\vec{r}_{i j}$ for all
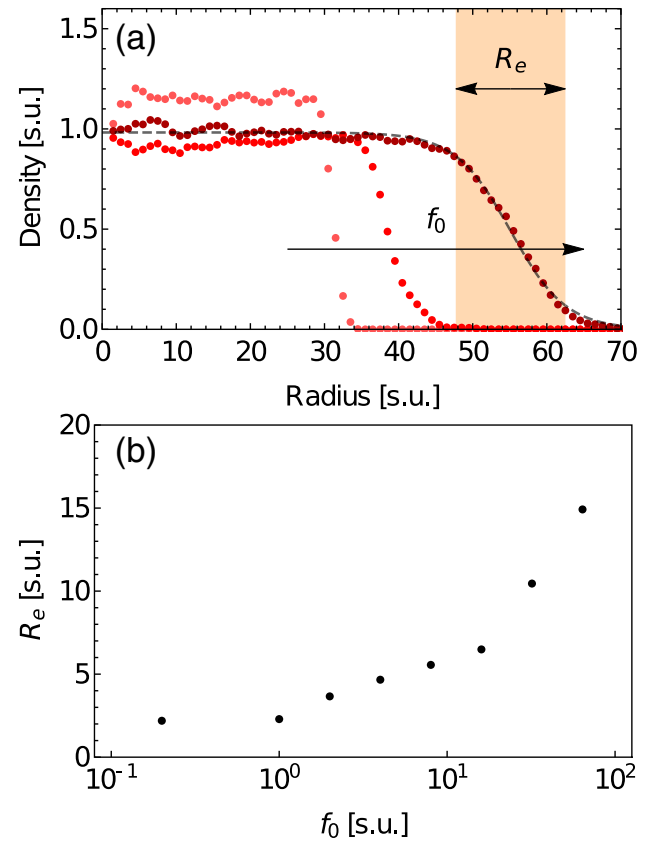

FIG. 16. Influence of the parameters $f_{0}$ on the shape of the density profile in simulations. (a) Density profiles for different values of $f_{0}$ at the same time step. From lightest to darkest: $f_{0}=0.2, f_{0}=16$, and $f_{0}=64$. All other parameters, and also the initial conditions, are identical. The black line on the dark red curve is a fit using a hyperbolic tangent, which allows us to extract the size of the edge (orange box). Note that, contrary to the results displayed in Fig. 3, no constraint on the velocity of cells at the border has been applied; therefore, the $C_{2}$ and $C_{3}$ compartments are not reproduced. (b) Size of the edge $R_{e}$ as a function of the parameter $f_{0}$ in the semilogarithmic scale. The value of the active force strength $\Gamma$ is identical for all cells and is set to 0.5 for each graph. cells. At each time step, all cells have the following division probability:

$$
\mathcal{P}= \begin{cases}0 & \text { if }\left|\vec{r}_{i j}\right|<R_{c, 1} \\ \frac{\left|\vec{r}_{i j}\right|-R_{c, 1}}{R_{c, 2}-R_{c, 1}} & \text { if } R_{c, 1}<\left|\vec{r}_{i j}\right|<R_{c, 2} \\ 1 & \text { if }\left|\vec{r}_{i j}\right|>R_{c, 2} .\end{cases}
$$

When division occurs, the two daughter cells inherit one building particle of their mother. Their second building particle is created at a distance $r_{c}$, with random uniform orientation with respect to the first building particle. The velocity of the daughter cells' building particle is $(1 / \sqrt{2})$ times the velocity of their mother's counterparts.

The shape of the cell is based on the Voronoi tessellation of the plane, using the particle nuclei position as the generating point. Given the positions $\vec{r}_{1}$ and $\vec{r}_{2}$ of the building points, the nuclei position is defined as $\vec{r}_{n}=\left(\vec{r}_{1}+\vec{r}_{2}\right) / 2$. The algorithm is hand-made. This technique allows for the extraction of the cell area and cell perimeter (for the associated formula, see Ref. [38]).

Finally, in Table III, we give the value of the parameters used in the simulations, with the exception of $\Gamma$ (see $\vec{F}_{a}$ )
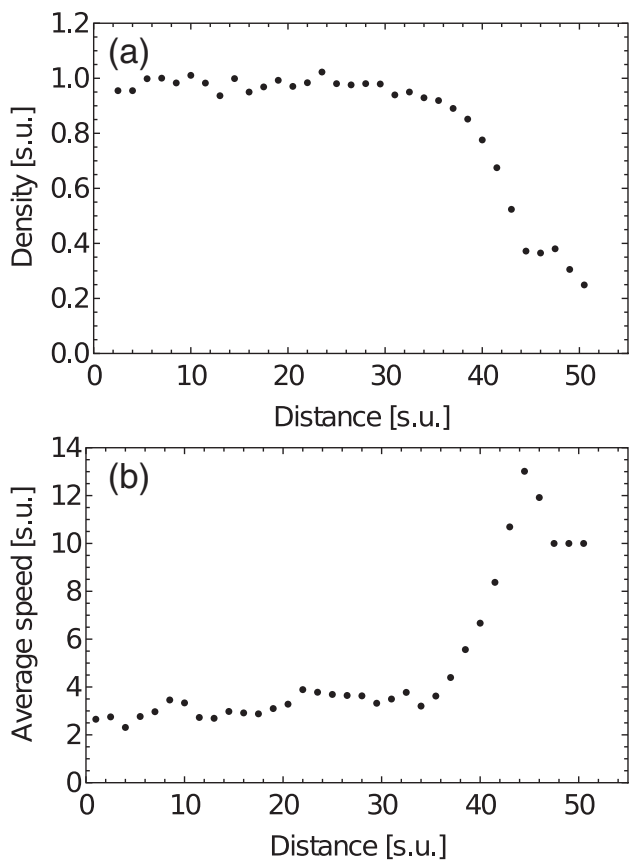

FIG. 17. Compartmentalization in the colony as emerging in the simulations, analogous to the glass substrate. In simulations, by externally saturating the cell speed in a ring at the periphery of the tissue, a local modulation of the cell density is seen at the edge. (a) Modulation of the density and apparition of the $C_{2}$ and $C_{3}$ compartments at the tissue edge. (b) Cell speed in the tissue and saturation. The saturation is set to $10 \mathrm{~s}$.u. in a 5 s.u. thick ring. The value of the active force strength $\Gamma$ is identical for all cells and is set to 0.5 for each graph, as in Fig. 3(b), mimicking the case on glass. 
and $f_{0}$ (see $\vec{F}_{c}$ ), which are varied in this article. In Fig. 16, we also provide an analysis of the influence of the parameter $f_{0}$ on the shape of the density profile, and in Fig. 17, we give a comparison between the speed profile and density profiles obtained from simulations when a constraint on the speed is applied. Here, we choose a speed saturation of 10 s.u. in a 5 s.u. thick ring at the edge of the tissue.

\section{APPENDIX F: DEFECTS IN MONOLAYERS}

As the tissues mature, different defects can be found in the monolayer, which are intrinsic features of the homeostatic state. Present on all substrates and the first to appear are scars associated with extrusion events (Fig. 18), in which cells are ejected from the tissue. The characteristic size of these defects is $20-40 \mu \mathrm{m}$. The tissue repairs these scars, often by division of neighboring cells.

About 8 days after seeding, cells deposit a significant amount of their own extracellular matrix, which induces local inhomogeneities in adhesiveness and the appearance of different types of defects on the monolayer in the central (oldest) compartment of the colony. Furthermore, we observe a drop of the average density and significant inhomogeneity in cell and nuclei structures.

On glass, a prominent type of defect is a dome, which emerges because of the physiological role of the MDCKII cells to pump from the apical to the basal side. On solid substrates (i.e., glass), this induces blisters in the tissues due to the accumulation of ions below the basal side [63]. Domes appear in large quantities after the homeostatic state is achieved. However, these are not permanent structures; after some time, they collapse back to the surface, and the comprising cells adhere again [see Figs. 19(a) and 19(b)]. The size of the domes, their lifetime, and frequency of occurrence [Fig. 19(c)] are

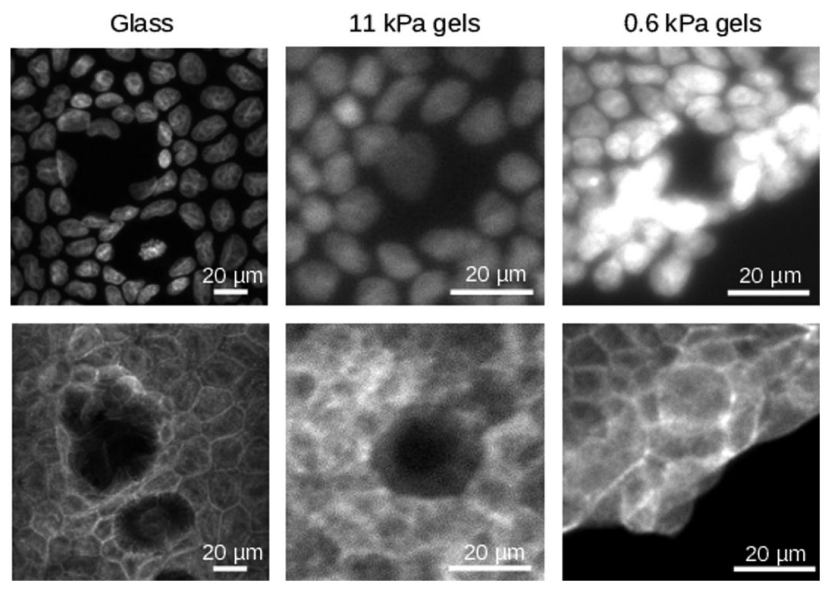

FIG. 18. Scars left by extrusion events in the tissue. We show pictures of scars left by extrusion seen through nuclei pictures (top) and actin pictures (bottom) for cells grown on glass, hard $11 \mathrm{kPa}$ gels, and soft $0.6 \mathrm{kPa}$ gels. (a)
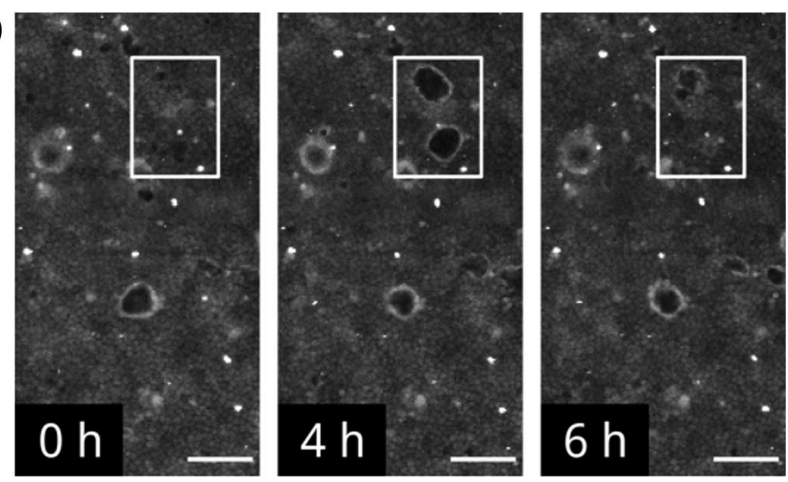

(b)

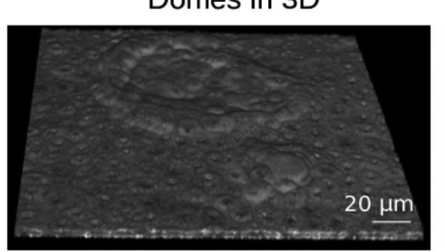

Basal side

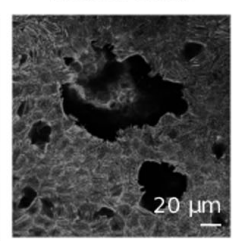

(c)
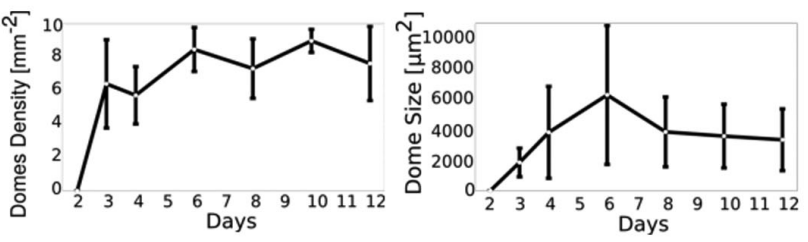

FIG. 19. Domes formation within the epithelial tissue. (a) Creation and dissolution of domelike structures in the bulk of mature MDCK-II monolayers. At $0 \mathrm{~h}$, all cells are in contact with the surface. At $4 \mathrm{~h}$, the domes are formed in the middle of the field of view. The cells are moved upwards and away from the focal plane. Only the base of the domes is visible, in the form of two bright circular structures. At $6 \mathrm{~h}$, the circular structures disappear as the domes collapse and the cells return to the focal plane. All scale bars account for $100 \mu \mathrm{m}$. (b) 3D structure of the domes and corresponding magnified picture of the basal side of the monolayer. (c) Long-term characterization of the domes' average density within the monolayer and size.

related to the content of the medium. In the conditions used in the current experiments, the characteristic size is $4000 \mu \mathrm{m}^{2}$.

[1] I. G. Macara, R. Guyer, G. Richardson, Y. Huo, and S. M. Ahmed, Epithelial Homeostasis, Curr. Biol. 24, R815 (2014).

[2] C.-P. Heisenberg and Y. Bellaïche, Forces in Tissue Morphogenesis and Patterning, Cell 153, 948 (2013).

[3] D. Krndija, F. El Marjou, B. Guirao, S. Richon, O. Leroy, Y. Bellaiche, E. Hannezo, and D. M. Vignjevic, Active cell migration is critical for steady-state epithelial turnover in the gut, Science 365, 705 (2019).

[4] A. I. McClatchey and A. S. Yap, Contact Inhibition (of Proliferation) Redux, Curr. Opin. Cell Biol. 24, 685 (2012).

[5] J. Zimmermann, B. A. Camley, W.-J. Rappel, and H. Levine, Contact Inhibition of Locomotion Determines 
Cell-Cell and Cell-Substrate Forces in Tissues, Proc. Natl. Acad. Sci. U.S.A. 113, 2660 (2016).

[6] Y. Gu, T. Forostyan, R. Sabbadini, and J. Rosenblatt, Epithelial Cell Extrusion Requires the Sphingosine-1Phosphate Receptor 2 Pathway, J. Cell Biol. 193, 667 (2011).

[7] B. Biteau, C. E. Hochmuth, and H. Jasper, Maintaining Tissue Homeostasis: Dynamic Control of Somatic Stem Cell Activity, Cell Stem Cell 9, 402 (2011).

[8] D. Hanahan and R. A. Weinberg, Hallmarks of Cancer: The Next Generation, Cell 144, 646 (2011).

[9] T. Das, K. Safferling, S. Rausch, N. Grabe, H. Boehm, and J. P. Spatz, A Molecular Mechanotransduction Pathway Regulates Collective Migration of Epithelial Cells, Nat. Cell Biol. 17, 276 (2015).

[10] A. Elosegui-Artola, R. Oria, Y. Chen, A. Kosmalska, C. Pérez-González, N. Castro, C. Zhu, X. Trepat, and P. Roca-Cusachs, Mechanical Regulation of a Molecular Clutch Defines Force Transmission and Transduction in Response to Matrix Rigidity, Nat. Cell Biol. 18, 540 (2016).

[11] J. Z. Kechagia, J. Ivaska, and P. Roca-Cusachs, Integrins as Biomechanical Sensors of the Microenvironment, Nat. Rev. Mol. Cell Biol. 20, 457 (2019).

[12] C. J. Miller and L. A. Davidson, The Interplay between Cell Signalling and Mechanics in Developmental Processes, Nat. Rev. Genet. 14, 733 (2013).

[13] M. Krausova and V. Korinek, Signal Transduction Pathways Participating in Homeostasis and Malignant Transformation of the Intestinal Tissue, Neoplasma 59, 708 (2012).

[14] E. Hannezo, J. Prost, and J.-F. Joanny, Theory of Epithelial Sheet Morphology in Three Dimensions, Proc. Natl. Acad. Sci. U.S.A. 111, 27 (2014).

[15] E. Paluch and C.-P. Heisenberg, Biology and Physics of Cell Shape Changes in Development, Curr. Biol. 19, R790 (2009).

[16] C. E. Chan and D. J. Odde, Traction Dynamics of Filopodia on Compliant Substrates, Science 322, 1687 (2008).

[17] K. Pogoda and P. A. Janmey, Glial Tissue Mechanics and Mechanosensing by Glial Cells, Front. Cell. Neurosci. 12, 25 (2018).

[18] K. Pogoda, R. Bucki, F. J. Byfield, K. Cruz, T. Lee, C. Marcinkiewicz, and P. A. Janmey, Soft Substrates Containing Hyaluronan Mimic the Effects of Increased Stiffness on Morphology, Motility, and Proliferation of Glioma Cells, Biomacromolecules 18, 3040 (2017).

[19] K. Pogoda, L. Chin, P. C. Georges, F. J. Byfield, R. Bucki, R. Kim, M. Weaver, R. G. Wells, C. Marcinkiewicz, and P. A. Janmey, Compression Stiffening of Brain and Its Effect on Mechanosensing by Glioma Cells, New J. Phys. 16, 075002 (2014).

[20] J. P. Califano and C. A. Reinhart-King, Substrate Stiffness and Cell Area Predict Cellular Traction Stresses in Single Cells and Cells in Contact, Cell. Mol. Bioeng. 3, 68 (2010).

[21] A. Zemel, F. Rehfeldt, A. E. X. Brown, D. E. Discher, and S. A. Safran, Optimal Matrix Rigidity for Stress-Fibre Polarization in Stem Cells, Nat. Phys. 6, 468 (2010).

[22] Y. Shao, K. Taniguchi, K. Gurdziel, R. F. Townshend, X. Xue, K. M. A. Yong, J. Sang, J. R. Spence, D. L. Gumucio, and J. Fu, Self-Organized Amniogenesis by Human
Pluripotent Stem Cells in a Biomimetic Implantation-like Niche, Nat. Mater. 16, 419 (2017).

[23] Y. Zheng, X. Xue, Y. Shao, S. Wang, S. N. Esfahani, Z. Li, J. M. Muncie, J. N. Lakins, V. M. Weaver, D. L. Gumucio, and J. Fu, Controlled Modelling of Human Epiblast and Amnion Development Using Stem Cells, Nature (London) 573, 421 (2019).

[24] M. C. Lampi and C. A. Reinhart-King, Targeting Extracellular Matrix Stiffness to Attenuate Disease: From Molecular Mechanisms to Clinical Trials, Sci. Transl. Med. 10, eaao0475 (2018).

[25] A. V. Taubenberger, S. Girardo, N. Träber, E. FischerFriedrich, M. Kräter, K. Wagner, T. Kurth, I. Richter, B. Haller, M. Binner, D. Hahn, U. Freudenberg, C. Werner, and J. Guck, 3D Microenvironment Stiffness Regulates Tumor Spheroid Growth and Mechanics via 21 and ROCK, Adv. Biosyst. 3, 1900128 (2019).

[26] Z. Gong, S. E. Szczesny, S. R. Caliari, E. E. Charrier, O. Chaudhuri, X. Cao, Y. Lin, R. L. Mauck, P. A. Janmey, J. A. Burdick, and V. B. Shenoy, Matching Material and Cellular Timescales Maximizes Cell Spreading on Viscoelastic Substrates, Proc. Natl. Acad. Sci. U.S.A. 115, E2686 (2018).

[27] E. E. Charrier, K. Pogoda, R. G. Wells, and P. A. Janmey, Control of Cell Morphology and Differentiation by Substrates with Independently Tunable Elasticity and Viscous Dissipation, Nat. Commun. 9, 449 (2018).

[28] B. F. Matte, A. Kumar, J. K. Placone, V. G. Zanella, M. D. Martins, A. J. Engler, and M. L. Lamers, Matrix Stiffness Mechanically Conditions EMT and Migratory Behavior of Oral Squamous Cell Carcinoma, J. Cell Sci. 132, jes224360 (2019).

[29] O. V. Sazonova, K. L. Lee, B. C. Isenberg, C. B. Rich, M. A. Nugent, and J. Y. Wong, Cell-Cell Interactions Mediate the Response of Vascular Smooth Muscle Cells to Substrate Stiffness, Biophys. J. 101, 622 (2011).

[30] W.-H. Guo, M. T. Frey, N. A. Burnham, and Y.-L. Wang, Substrate Rigidity Regulates the Formation and Maintenance of Tissues, Biophys. J. 90, 2213 (2006).

[31] X. Trepat, M. R. Wasserman, T. E. Angelini, E. Millet, D. A. Weitz, J.P. Butler, and J. J. Fredberg, Physical Forces During Collective Cell Migration, Nat. Phys. 5, 426 (2009).

[32] T. Yeung, P. C. Georges, L. A. Flanagan, B. Marg, M. Ortiz, M. Funaki, N. Zahir, W. Ming, V. Weaver, and P. A. Janmey, Effects of Substrate Stiffness on Cell Morphology, Cytoskeletal Structure, and Adhesion, Cell Motil. Cytoskeleton 60, 24 (2005).

[33] J. L. Leight, M. A. Wozniak, S. Chen, M. L. Lynch, and C. S. Chen, Matrix Rigidity Regulates a Switch between TGF- $\beta 1$-Induced Apoptosis and Epithelial-Mesenchymal Transition, Mol. Biol. Cell 23, 781 (2012).

[34] S. Kaliman, C. Jayachandran, F. Rehfeldt, and A.-S. Smith, Novel Growth Regime of MDCK II Model Tissues on Soft Substrates, Biophys. J. 106, L25 (2014).

[35] D. T. Tambe, C. C. Hardin, T. E. Angelini, K. Rajendran, C. Y. Park, X. Serra-Picamal, E. H. Zhou, M. H. Zaman, J. P. Butler, D. A. Weitz, J. J. Fredberg, and X. Trepat, Collective Cell Guidance by Cooperative Intercellular Forces, Nat. Mater. 10, 469 (2011). 
[36] A. Ravasio, A. P. Le, T. B. Saw, V. Tarle, H. T. Ong, C. Bertocchi, R.-M. Mège, C. T. Lim, N. S. Gov, and B. Ladoux, Regulation of Epithelial Cell Organization by Tuning Cell-Substrate Adhesion, Integr. Biol. 7, 1228 (2015).

[37] K. Kolahi, P. White, D. Shreter, A.-K. Classen, D. Bilder, and M. Mofrad, Quantitative Analysis of Epithelial Morphogenesis in Drosophila Oogenesis: New Insights Based on Morphometric Analysis and Mechanical Modeling, Dev. Biol. 331, 129 (2009).

[38] S. Kaliman, C. Jayachandran, F. Rehfeldt, and A.-S. Smith, Limits of Applicability of the Voronoi Tessellation Determined by Centers of Cell Nuclei to Epithelium Morphology, Front. Physiol. 7, 551 (2016).

[39] S. A. Sandersius, M. Chuai, C. J. Weijer, and T. J. Newman, Correlating Cell Behavior with Tissue Topology in Embryonic Epithelia, PLoS One 6, e18081 (2011).

[40] J. Lovrić, S. Kaliman, W. Barfuss, G. E. Schröder-Turk, and A.-S. Smith, Geometric Effects in Random Assemblies of Ellipses, Soft Matter 15, 8566 (2019).

[41] D. Aboav, The Arrangement of Grains in a Polycrystal, Metallography 3, 383 (1970).

[42] D. Aboav, The Arrangement of Cells in a Net, Metallography 13, 43 (1980).

[43] D. Weaire, Some Remarks on the Arrangement of Grains in a Polycrystal, Metallography 7, 157 (1974).

[44] G. L. Caer and R. Delannay, Correlations in Topological Models of 2D Random Cellular Structures, J. Phys. A 26, 3931 (1993).

[45] G. Vincze, I. Zsoldos, and A. Szasz, On the Aboav-Weaire Law, J. Geom. Phys. 51, 1 (2004).

[46] J. Saraiva, P. Pina, L. Bandeira, and J. Antunes, Polygonal Networks on the Surface of Mars; Applicability of Lewis, Desch and Aboav-Weaire Laws, Philos. Mag. Lett. 89, 185 (2009).

[47] J. C. M. Mombach, M. A. Z. Vasconcellos, and R. M. C. de Almeida, Arrangement of Cells in Vegetable Tissues, J. Phys. D 23, 600 (1990).

[48] D. Wenzel, S. Praetorius, and A. Voigt, Topological and Geometrical Quantities in Active Cellular Structures, J. Chem. Phys. 150, 164108 (2019).

[49] F. T. Lewis, A Comparison between the Mosaic of Polygons in a Film of Artificial Emulsion and the Pattern of Simple Epithelium in Surface View (Cucumber Epidermis and Human Amnion), Anat. Rec. 50, 235 (1931).

[50] F. T. Lewis, The Correlation between Cell Division and the Shapes and Sizes of Prismatic Cells in the Epidermis of Cucumis, Anat. Rec. 38, 341 (1928).

[51] S. N. Chiu, Aboav-Weaire's and Lewis' Laws-A Review, Mater. Charact. 34, 149 (1995).

[52] S. Kim, M. Cai, and S. Hilgenfeldt, Lewis' Law Revisited: The Role of Anisotropy in Size-Topology Correlations, New J. Phys. 16, 015024 (2014).

[53] N. Rivier, Statistical Crystallography Structure of Random Cellular Networks, Philos. Mag. B 52, 795 (1985).

[54] D. Bi, J. H. Lopez, J. M. Schwarz, and M. L. Manning, A Density-Independent Rigidity Transition in Biological Tissues, Nat. Phys. 11, 1074 (2015).
[55] M. Vishwakarma, J. P. Spatz, and T. Das, Mechanobiology of Leader-Follower Dynamics in Epithelial Cell Migration, Curr. Opin. Cell Biol. 66, 97 (2020).

[56] M. Basan, J. Prost, J.-F. Joanny, and J. Elgeti, Dissipative Particle Dynamics Simulations for Biological Tissues: Rheology and Competition, Phys. Biol. 8, 026014 (2011).

[57] N. Ganai, T. Büscher, G. Gompper, and J. Elgeti, Mechanics of Tissue Competition: Interfaces Stabilize Coexistence, New J. Phys. 21, 063017 (2019).

[58] J. Ranft, M. Basan, J. Elgeti, J.-F. Joanny, J. Prost, and F. Julicher, Fluidization of Tissues by Cell Division and Apoptosis, Proc. Natl. Acad. Sci. U.S.A. 107, 20863 (2010).

[59] S. Tlili, E. Gauquelin, B. Li, O. Cardoso, B. Ladoux, H. Delanoë-Ayari, and F. Graner, Collective Cell Migration without Proliferation: Density Determines Cell Velocity and Wave Velocity, R. Soc. Open Sci. 5, 172421 (2018).

[60] P. A. Janmey, D. A. Fletcher, and C. A. Reinhart-King, Stiffness Sensing by Cells, Physiol. Rev. 100, 695 (2020).

[61] G. Peyret, R. Mueller, J. D’Alessandro, S. Begnaud, P. Marcq, R. M. Mège, J. M. Yeomans, A. Doostmohammadi, and B. Ladoux, Sustained Oscillations of Epithelial Cell Sheets, Biophys. J. 117, 464 (2019).

[62] E. Marinari, A. Mehonic, S. Curran, J. Gale, T. Duke, and B. Baum, Live-Cell Delamination Counterbalances Epithelial Growth to Limit Tissue Overcrowding, Nature (London) 484, 542 (2012).

[63] E. Latorre, S. Kale, L. Casares, M. Gómez-González, M. Uroz, L. Valon, R. V. Nair, E. Garreta, N. Montserrat, A. del Campo, B. Ladoux, M. Arroyo, and X. Trepat, Active Superelasticity in Three-Dimensional Epithelia of Controlled Shape, Nature (London) 563, 203 (2018).

[64] F. Rehfeldt, A. E. X. Brown, M. Raab, S. Cai, A. L. Zajac, A. Zemel, and D. E. Discher, Hyaluronic Acid Matrices Show Matrix Stiffness in 2D and 3D Dictates Cytoskeletal Order and Myosin-II Phosphorylation within Stem Cells, Integr. Biol. 4, 422 (2012).

[65] B. G. Lorz, A.-S. Smith, C. Gege, and E. Sackmann, Adhesion of Giant Vesicles Mediated by Weak Binding of Sialyl-Lewis $X$ to E-Selectin in the Presence of Repelling Poly(ethylene glycol) Molecules, Langmuir 23, 12293 (2007).

[66] I. Levental, P. C. Georges, and P. A. Janmey, Soft Biological Materials and Their Impact on Cell Function, Soft Matter 3, 299 (2007).

[67] E. Garreta, P. Prado, C. Tarantino, R. Oria, L. Fanlo, E. Martí, D. Zalvidea, X. Trepat, P. Roca-Cusachs, A. GavaldàNavarro, L. Cozzuto, J. M. Campistol, J. C. I. Belmonte, C. H. del Pozo, and N. Montserrat, Fine Tuning the Extracellular Environment Accelerates the Derivation of Kidney Organoids from Human Pluripotent Stem Cells, Nat. Mater. 18, 397 (2019).

[68] S. Nehls, H. Nöding, S. Karsch, F. Ries, and A. Janshoff, Stiffness of MDCK II Cells Depends on Confluency and Cell Size, Biophys. J. 116, 2204 (2019).

[69] K. Doxzen, S. R. K. Vedula, M. C. Leong, H. Hirata, N. S. Gov, A. J. Kabla, B. Ladoux, and C. T. Lim, Guidance of Collective Cell Migration by Substrate Geometry, Integr. Biol. (United Kingdom) 5, 1026 (2013). 
[70] M. A. Klatt, J. Lovrić, D. Chen, S. C. Kapfer, F. M. Schaller, P. W. A. Schönhöfer, B. S. Gardiner, A.-S. Smith, G. E. Schröder-Turk, and S. Torquato, Universal Hidden Order in Amorphous Cellular Geometries, Nat. Commun. 10, 811 (2019).

[71] A. Engler, L. Bacakova, C. Newman, A. Hategan, M. Griffin, and D. Discher, Substrate Compliance versus Ligand Density in Cell on Gel Responses, Biophys. J. 86, 617 (2004).

[72] A. J. Engler, S. Sen, H. L. Sweeney, and D. E. Discher, Matrix Elasticity Directs Stem Cell Lineage Specification, Cell 126, 677 (2006).

[73] S. Kaliman, Ph.D. thesis, Friedrich-Alexander-Universität Erlangen-Nürnberg (2018).
[74] S. Preibisch, S. Saalfeld, and P. Tomancak, Globally Optimal Stitching of Tiled 3D Microscopic Image Acquisitions, Bioinformatics 25, 1463 (2009).

[75] Q. Tseng, E. Duchemin-Pelletier, A. Deshiere, M. Balland, H. Guillou, O. Filhol, and M. Thery, Spatial Organization of the Extracellular Matrix Regulates Cell-Cell Junction Positioning, Proc. Natl. Acad. Sci. U.S.A. 109, 1506 (2012).

Correction: A proof change request was misinterpreted by the production team and resulted in a duplication of Affiliation 7 and in the disappearance of Affiliation 8. Both problems have been fixed. 